\title{
Arc/Arg3.1 mRNA global expression patterns elicited by memory recall in cerebral cortex differ for remote versus recent spatial memories
}

\author{
Pavel A. Gusev ${ }^{*}$ and Alexander N. Gubin ${ }^{2}$ \\ Blanchette Rockefeller Neurosciences Institute, Rockville, MD, USA \\ 2 Laboratory of Developmental Biology, Food and Drug Administration, Bethesda, MD, USA
}

\section{Edited by:}

Rui M. Costa, Instituto Gulbenkian de

Ciência, Portugal

\section{Reviewed by:}

PaulW. Frankland, Hospital for Sick

Children, Canada;

University of Toronto, Canada

Kuan H. Wang, National Institutes of

Health, USA

\section{${ }^{*}$ Correspondence:}

Pavel A. Gusev, Department of

Pharmacology, Uniformed Services

University of Health Sciences, 4301

Jones Bridge Road, Bethesda, MD

20814, USA.

e-mail:pgusev@usuhs.mil
The neocortex plays a critical role in the gradual formation and storage of remote declarative memories. Because the circuitry mechanisms of systems-level consolidation are not well understood, the precise cortical sites for memory storage and the nature of enduring memory correlates (mnemonic plasticity) are largely unknown. Detailed maps of neuronal activity underlying recent and remote memory recall highlight brain regions that participate in systems consolidation and constitute putative storage sites, and thus may facilitate detection of mnemonic plasticity. To localize cortical regions involved in the recall of a spatial memory task, we trained rats in a water-maze and then mapped mRNA expression patterns of a neuronal activity marker Arcl Arg3. 1 (ArC) upon recall of recent (24 h after training) or remote (1 month after training) memories and compared them with swimming and naive controls. Arc gene expression was significantly more robust $24 \mathrm{~h}$ after training compared to 1 month after training. Arc expression diminished in the parietal, cingulate and visual areas, but select segments in the prefrontal, retrosplenial, somatosensory and motor cortical showed similar robust increases in the Arc expression. When Arc expression was compared across select segments of sensory, motor and associative regions within recent and remote memory groups, the overall magnitude and cortical laminar patterns of task-specific Arc expression were similar (stereotypical). Arc mRNA fractions expressed in the upper cortical layers $(2 / 3,4)$ increased after both recent and remote recall, while layer 6 fractions decreased only after the recent recall. The data suggest that robust recall of remote memory requires an overall smaller increase in neuronal activity within fewer cortical segments. This activity trend highlights the difficulty in detecting the storage sites and plasticity underlying remote memory. Application of the Arc maps may ameliorate this difficulty.

Keywords: rat, immediate-early gene, neocortex, stereotypy, water maze

\section{INTRODUCTION}

The representation of declarative memories in the brain determined by mapping, lesion and inactivation studies changes over the time (Frankland and Bontempi, 2005; Squire and Bayley, 2007). While the hippocampus plays a critical role in memory acquisition and its early retention, or may even be persistently involved in memory representation (for reference see Lehmann et al., 2007), the neocortex is critical for the gradual formation and further storage of remote memories. This transition in memory representation reflects systems-level memory consolidation the mechanisms of which are largely unknown.

For a spatial memory task such as the water-maze (WM), the expansion of memory representation into the neocortex occurs within 7-10 days after completion of training (Frankland et al.,

Abbreviations: WM, water-maze; WMT, water-maze trained; SW, swimming control; IEG, immediate-early gene; Arc, activity-regulated cytoskeleton-associated; $\mathrm{Cg} 1$ and $\mathrm{Cg} 2$, cingulate cortex 1 and 2; RS, retrosplenial, Ins, insular cortices; $\mathrm{MO}$, medial orbital; VO, ventral orbital cortex; DLO, dorso-lateral orbital cortex; parietal association (Par), visual primary (V1) and secondary medial and lateral (V2M; V2L) cortices; somatosensory and motor primary and secondary cortices (S1, S2, M1, M2, respectively); FrAs, frontal association; prelimbic (Prl) and lateral orbital (LO) cortices; LEA, lateral entorhinal area; L, layer; ROI, region of interest.
2001; Remondes and Schuman, 2004; Teixeira et al., 2006). It is believed that enduring neuronal plasticity underlies long-term memory storage (mnemonic plasticity; Kim and Linden, 2007). The neocortical CaMKII expression related to synaptic plasticity is critical for remote spatial memory formation (Frankland et al., 2001; Tanaka et al., 2008). However the exact nature of enduring functional and structural neuronal correlates for remote WM memories remains undetermined. Sparse information representation in the brain (Laughlin and Sejnowski, 2003; Waydo et al., 2006) and lack of detailed knowledge of cortical circuitries underlying remote memory make it difficult to detect sparsely distributed enduring memory correlates (Xu et al., 2009; Yang et al., 2009). On the other hand, neurons active during memory recall might highlight storages sites that express mnemonic plasticity. Thus, the stereotypical or well-ordered organization of memory-related activity could facilitate detection of enduring storage correlates because it could point to the most common storage sites across the entire cortex.

Microcircuits across the entire neocortex are characterized by a number of common types of neurons connected in similar patterns (Silberberg et al., 2002; Mountcastle, 2003; Thomson and Bannister, 2003; Markram, 2006). Moreover, similar aspects in 
interlaminar transformation of neuronal responses to sensory stimuli of different modalities have also been described (Hirsch and Martinez, 2006). In addition, patterns of gene expression in cortical layers repeat themselves across the entire neocortex (Lein et al., 2007). This type of consistent organization has been termed stereotypy (Silberberg et al., 2002). However, it is unknown to what degree memory-related activity in the cortex has a stereotypical organization. Would changes in the magnitude of neuronal activity and its laminar distribution specific to a memory recall be similar across the subset of activated cortical areas? What is the relationship between the stereotypy and memory consolidation?

Compared to the hippocampus, the laminar organization of the cerebral cortex is more complex but the anatomical structure in both regions is somewhat repetitive (Amaral and Witter, 1989). To better understand the circuitries underlying systems-level consolidation, we previously constructed detailed maps of memoryrelated activity in the hippocampus (Gusev et al., 2005). We used Arc/Arg3.1 mRNA expression as a molecular marker for neuronal activity induced by recent and remote WM memory recall. Arc/ Arg3.1 is an effector immediate-early gene (IEG) encoding an activity-regulated cytoskeleton-associated $(A r c)$ and a synaptic protein expressed by cortical excitatory neurons following strong synaptic activity and behavioral experiences (Link et al., 1995; Lyford et al., 1995; Guzowski et al., 2000; Vazdarjanova et al., 2006). Arc has been implicated in Hebbian and homeostatic neuronal plasticity, memory consolidation, and experience-dependent changes in sensory representations (Guzowski et al., 2000; Plath et al., 2006; Rial Verde et al., 2006; Wang et al., 2006). Moreover, our detection and mapping studies suggest a link between mnemonic plasticity recorded in CA1 pyramidal cells (such as disinhibition and larger spike amplitude) and an increased Arc expression in CA1 after recent memory recall (Gusev and Alkon, 2001; Gusev et al., 2005). An intriguing possibility would be that Arc expression induced by remote recall indicates neurons expressing mnemonic plasticity underlying permanent storage.

In agreement with a persistent role of the hippocampus in WM memory, we previously found that both recent and remote recall of WM memory induces more Arc mRNA expression in hippocampal regions when compared to swimming controls (Gusev et al., 2005). However, after a 1-month retention interval, the $\operatorname{Arc}$ mRNA task-specific expression reorganizes and becomes smaller and clusters within only select segments of the hippocampus. We now use the same behavioral paradigm to establish global changes in neocortical activity during remote memory formation.

We tested the hypothesis that recent memory recall induces robust and stereotypical increase in Arc expression widely distributed across the entire cortex while remote recall induces less Arc expression clustered in select cortical segments and layers. We found that the main trend in global reorganization of neuronal activity induced by memory recall in the cerebral cortex parallels the changes we described previously for the hippocampus (Gusev et al., 2005). The Arc maps characterize the outcome of systems-level consolidation and highlight cortical regions and layers participating in remote memory formation and storage. These maps may facilitate detection of enduring neuronal correlates of memory storage.

\section{MATERIALS AND METHODS TRAINING PROCEDURE}

The subjects were 30 8-week-old male Wistar rats (Charles River Laboratory), the same as those used for hippocampal activity mapping in our previous study (Gusev et al., 2005). Rats were individually caged, given access to food and water, and maintained on a 12:12-h light/dark cycle. Animal care and maintenance was in accordance with NIH guidelines. Rats were trained in a water-maze task in a swimming pool $1.5 \mathrm{~m}$ in diameter and $0.6 \mathrm{~m}$ high filled with milky water $\left(24 \pm 1^{\circ} \mathrm{C}\right)$ and located in a well-lit $(3 \mathrm{~m} \times 5 \mathrm{~m})$ room with distinct extra-maze cues. Our training protocol was designed to force rats to use a spatial navigation strategy (Morris, 1984; Eichenbaum et al., 1990; Micheau et al., 2004). A transparent square platform $(12.5 \mathrm{~cm} \times 12.5 \mathrm{~cm})$ was hidden in the same location (quadrant's center) within the pool with its top surface submerged $1.5 \mathrm{~cm}$ below the water level. On day 0 , rats were subjected to $2.5 \mathrm{~min}$ of swimming in the pool in the absence of the platform, for adaptation to the environment. On the first day, an animal was guided to the platform if it did not find it within $2 \mathrm{~min}$. For the next 7 days, rats were trained to locate the hidden platform on four trials per day. Rats were brought into the training room in a home cage one at a time. After holding the rat for $1 \mathrm{~min}$, a mark was applied to the head with a wide tip black marker to enable tracking using a PolyTrack video system (San Diego Instruments Inc., CA, USA). Before placing the rat into the tank to begin training trials, the rat was carried around the tank twice within 2 min with 10 -s stops at the quadrant's borders. These walks were introduced to extend context exposure and further facilitate memory recall especially after a 1-month delay. A rat was placed into the water facing the tank's wall at the middle of quadrant opposite, adjacent left and right of the target quadrant. Rats were then allowed to stay on the platform for $40 \mathrm{~s}$. After each trial, the experimenter walked away from the pool and started the next trial with a 10-s delay from a different quadrant. The order of the starting positions was pseudo-randomized and varied every day. After four trials were completed, the rat was placed into a cage filled with clear water for 5-10 s to rinse the milk, and then it was placed into a cardboard box filled with wood chips for $20 \mathrm{~s}$ to let it shake off water. The rat was then toweled before being returned to the home cage. For all animals, there was only one target quadrant and it was always in the same location. Acquisition of spatial memory was assessed based on escape latencies. Two out of five rats from 1-month group were given one extra training session.

In order to control for non-mnemonic and mnemonic processes not specifically related to our navigational task, we used animal triads that included one swim control (SW) and one naïve control for each water-maze trained (WMT) rat. In order to equate locomotor activity and stress responses, rats from the SW control group had one trial of swimming each day. The time of the control swim was yoked to the time required to find the platform for the corresponding WMT rat on that training day. Swimming controls spent $3 \mathrm{~min}$ in the cardboard box to equate the time that WMT rats spent on the platform $(4 \times 40 \mathrm{~s})$. Five animal triads were used to map representation of recent memory recall and another five triads were used to study remote memory recall. 


\section{PROBE TESTS FOR BRAIN ACTIVITY MAPPING}

During the probe tests, the WMT and yoked SW animals were treated according to the protocol followed during the training sessions except that both groups spent $20 \mathrm{~s}$ in the cardboard box following the tests and before being returned to their home cage and the housing room. Three consecutive probe tests with 10 -s intervals were performed $24 \mathrm{~h}$ or 1 month after the last training session. After being released into the water, rats faced the wall in the middle of the quadrant opposite to the target quadrant location and swam for $60 \mathrm{~s}$ with the platform removed. Navigation skills were evaluated based on the number of "target area" $(12.5 \mathrm{~cm} \times 12.5 \mathrm{~cm})$ crossings, proximity to the platform location (Gallagher et al., 1993; Maei et al., 2009) and latency to the first target crossing. We also report results of quadrant based analyses of dwell times and distances (Figures S1 and S2 in Supplementary Material). Proximity was measured as the mean distance between a rat and the platform during a probe trial (in tracker units). Linear distance from the platform marker to a rat was measured on every video tracker frame (18/s). We conducted a comparative study of sensitivity and reliability of different parameters for WM task memory in our behavioral protocol and experimental set-up (see Supplementary Material).

The entire test procedure took about $8 \mathrm{~min}$, and was conducted on WMT and SW rats from each triad with a 10-min interval. Animals were killed $30 \mathrm{~min}$ following the end of the last probe test and brains were quickly removed from the skull and placed on dry ice powder within 3 min. Naïve controls were killed last in each triad, $15 \mathrm{~min}$ after the swim controls. A 30-min delay was chosen because at this point Arc signal is no longer present as nuclear foci (Guzowski et al., 1999; Gusev et al., unpublished observation). After Arc transcription has largely ended, the full magnitude of justgenerated Arc signal can be measured more precisely compared to earlier time points when transcription is still in progress. By measuring the Arc signal diffused within the soma we also avoid film saturation. This saturation may occur over a highly condensed Arc signal present in the foci. Therefore with a 30-min delay, differences in Arc signal magnitude should be better detected.

Remote memory recall may be a more difficult task compared to recent memory recall (Biedenkapp and Rudy, 2007; Wiltgen and Silva, 2007). To ensure robust memory recall at all time points after the training, we extended an animal's exposure to the training room context by carrying (twice for $1 \mathrm{~min}$; see above) a rat around the pool before the tests. We also used triplets of the probe test to give additional reminders of the task and to monitor possible timedependent changes in task performance over the three probes. This design applied to both the recent and remote memory groups and ensured clear behavioral responses and strong in situ hybridization signals. Under this schedule, the extinction of WM search behavior did not develop during the probes.

It is likely that some forms of learning related to the experience and the environment are common for SW control and WMT rats (Kubik et al., 2007). Employing such common skills could induce Arc expression in the both groups. This activity may also mask some aspects of Arc expression related to the WM task. The SW controls, however, were never trained to navigate to a submerged platform and thus do not recall relevant spatial memory. To balance the possible effects of stress on Arc expression in WMT animals due to absence of the platform, we elevated stress in SW controls by switching from a single daily swim during the training sessions to three swim trials during the probe tests as reported earlier (Gusev et al., 2005). Therefore, the SW control should help to dissect the Arc expression representing activation of navigation skills and recall of spatial memory. In other studies, animals trained on a visible platform task have also been successfully used as control for WM memory (Teixeira et al., 2006; Kee et al., 2007).

We designed the current behavioral and mapping protocols in order to study the effects of putative treatments for memory disorders and enhancement of memory ensembles. We avoided animal overtraining and create room for memory enhancement. Yet, we still induced robust memory retention over 1 month later. This approach will allow us to explore how brain regions mediate memory enhancement and its decline in models of memory disorders.

\section{CORTICOSTERONE MEASUREMENT}

Stress levels caused by behavioral procedures were measured by the concentration of corticosterone in the blood separately from the mapping triads. The stress triads were trained in a slightly larger tank (diameter, $180 \mathrm{~cm}$ ). Recent memory tests and control swimming procedures were analogous to those used in the mapping experiments. We also measured the effect of context exposure in the water-maze training room on corticosterone levels $24 \mathrm{~h}$ after the last training session. WMT and SW rats were brought into the room, and their heads were marked. Animals were held in an experimenter's hands during the two 1-min walks around the pool. Afterward, they were immediately placed into home cages and returned to the housing room, without starting a swimming session. Animals were taken from their home cages and killed in a procedure room $20 \mathrm{~min}$ after the context-only exposures or probe tests. WMT, SW, and naive rats from each triad were killed within an 80 -min time span to ensure the same phase of the circadian rhythm for the entire triad (i.e., between 9:30 A.M. and 3:00 P.M.). Trunk blood was collected into $1.5 \mathrm{ml}$ tubes on ice containing $5 \mu \mathrm{l}$ of $0.13 \mathrm{M}$ EDTA. Samples were centrifuged immediately at $4^{\circ} \mathrm{C}$ at $14,000 \mathrm{rpm}$ for $5 \mathrm{~min}$. Plasma aliquots were stored at $-80^{\circ} \mathrm{C}$. Corticosterone concentration was determined by an enzyme immunoassay kit with a 96-well microtiter coated with polyclonal antibody raised against corticosterone following the manufacturer's instructions (Immunodiagnostic Systems, Fountain Hills, AZ, USA). The absorbance levels were measured with a Model 550 microplate reader at $450 \mathrm{~nm}$ (reference at $655 \mathrm{~nm}$; Bio-Rad, Hercules, CA, USA).

\section{BRAIN SECTIONING}

Coronal $20-\mu \mathrm{m}$-thick cryostat sections were collected in a rostrocaudal direction across the whole brain. We used the interaural system of coordinates to measure and to assign coordinates to the brain sections during the forebrain sectioning, and then to create anatomical maps of neuronal activity. To obtain these coordinates, we needed to adjust the distance measured between the rostral pole of a frozen brain and the rear-most edge of the neocortex by adding $0.6 \mathrm{~mm}$. Due to small variations in brain size from animal to animal, we also compared the anatomy of sections with a rat brain atlas and made necessary adjustments before assigning coordinates (Paxinos and Watson, 1998). During the sectioning 
with a Leica CM 1900 cryostat (Leica Microsystems, Germany), the coordinates were calculated based on the number of $20-\mu \mathrm{m}$ sections. The progress of brain sectioning was also monitored with a compass and a ruler. There was good correspondence between the measured, calculated and anatomical coordinates during the sectioning. Brain sections on the same slide were separated by no less than $60 \mu \mathrm{m}$ to avoid measuring the Arc mRNA signal in the same neurons twice. During the sectioning, each section was placed on the next silanated glass slide (K-D Medical Inc., Columbia, MD, USA) out of a group of four slides. Three coronal sections were mounted per slide, (four sections were mounted for the very frontal areas). A protocol number of a behavioral group and an estimated interaural coordinate for the last mounted section on the fourth slide were written on each of four slides so the coordinate of each section could be calculated as precisely as possible. In total, 100 slides were collected per animal. During the cryostat sectioning, the slides were kept at room temperature in a slide container. After the sectioning was complete, tissue sections were stored at $-80^{\circ} \mathrm{C}$ until use.

\section{REVERSE TRANSCRIPTASE-POLYMERASE CHAIN REACTION AND Arc PROBE GENERATION}

Total rat RNA was purchased from Ambion (Austin, TX, USA). Reverse transcription (RT) was performed using the oligo-(dT) primer and Superscript Reverse Transcriptase at $42^{\circ} \mathrm{C}$ for $50 \mathrm{~min}$, as suggested by the manufacturer (Invitrogen, Carlsbad, CA, USA). We used $1 \mu \mathrm{l}$ RT reaction in $50 \mu$ l polymerase chain reaction (PCR) amplification with the appropriate primers as follows: initial denaturation at $95^{\circ} \mathrm{C}$ for $2 \mathrm{~min}$, denaturing at $94^{\circ} \mathrm{C}$ for $30 \mathrm{~s}$, annealing at $62^{\circ} \mathrm{C}$ for $30 \mathrm{~s}$, and extension at $72^{\circ} \mathrm{C}$ for $3 \mathrm{~min}$ for 36 cycles. For Arc amplification, 5'-ACGGGCGACTCACAGCGCTGGA-3' and 5'-GGGTCTCCTGGGACTGGACTTGA-CCA-3' primers were used (expected product size is $2.1 \mathrm{~Kb}$ ). The PCR product was analyzed on a $1.2 \%$ agarose gel stained with ethidium bromide (Invitrogen). The PCR product was subcloned into a pCR4-TOPO vector (Invitrogen) and the positive Arc clone was confirmed by restriction digestion and sequencing. Purified plasmid DNA was linearized either with PmeI or NotI (New England Biolabs, Beverly, MA, USA). Both linearized DNA templates were used to generate sense and antisense RNA probes labeled with ${ }^{35} \mathrm{~S}\left(\alpha-{ }^{35} \mathrm{~S}\right.$-UTP; Life Sciences Perkin-Elmer, Boston, MA, USA; Amersham Biosciences, Piscataway, NJ, USA) using Ambion's MAXIscript kit according to the manufacturer's instructions. The yield and integrity of riboprobe was verified by gel electrophoresis.

\section{IN SITUHYBRIDIZATION HISTOCHEMISTRY}

Experiments were performed with the brain sections taken at the same brain level from the WMT, SW and naïve control animals in parallel. An exact anatomical match of the sections was performed on autoradiograms. The sections were fixed in a buffered $(1 \times$ PBS) $4 \%$ formaldehyde solution freshly prepared from paraformaldehyde for $5 \mathrm{~min}$, and rinsed in PBS two times for $5 \mathrm{~min}$. Then slices were placed in $0.25 \%$ acetic anhydride in $0.1 \mathrm{M}$ triethanolamine for $10 \mathrm{~min}$, rinsed for $5 \mathrm{~min}$ in $2 \times$ standard saline citrate (SSC, $1 \times$ $\mathrm{SSC}=0.15 \mathrm{M} \mathrm{NaCl}$ : $0.015 \mathrm{M}$ sodium citrate), dehydrated by passing them for $1 \mathrm{~min}$ through graded dilutions of ethanol in distilled water (v/v) $(70 \%, 80 \%, 95 \%, 100 \%)$. Sections were passed through chloroform $\left(\mathrm{CHCl}_{3}\right)$ for $5 \mathrm{~min}$, ethanol $100 \%$ and $95 \%$ for $1 \mathrm{~min}$ in each and air-dried for $30 \mathrm{~min}$. The Arc riboprobe $\left(1 \times 10^{6} \mathrm{cpm}\right.$ in $50 \mu \mathrm{l})$ was applied to each slide holding three sections and hybridized at $55^{\circ} \mathrm{C}$ for $24 \mathrm{~h}$ in a mixture containing $20 \mathrm{mM}$ Tris- $\mathrm{HCl}, \mathrm{pH} 7.4$, $1 \mathrm{mM}$ EDTA, $300 \mathrm{mM} \mathrm{NaCl}, 50 \%$ formamide, $10 \%$ dextran sulfate, $1 \times$ Denhardt's solution, $4 \mu \mathrm{g} / \mathrm{ml}$ salmon sperm DNA, $10 \mu \mathrm{g} / \mathrm{ml}$ yeast total RNA, $10 \mu \mathrm{g} / \mathrm{ml}$ yeast tRNA (Invitrogen), $100 \mathrm{mM}$ dithiothreitol (ICN Biomedicals Inc, Irvine, CA, USA), 0.1\% SDS, $0.1 \%$ NTS. Compounds were purchased from Sigma-Aldrich Co. (St. Louis, MO, USA) unless otherwise mentioned. Slices hybridized with the sense riboprobe were used as negative controls. Glass coverslips were placed on hybridizing sections. After high stringency posthybridization washes and RNase treatment, brain sections were dehydrated in graded ethanol and then subjected to autoradiography with BioMax MR film (Eastman Kodak Company, Rochester, NY, USA) together with ${ }^{14} \mathrm{C}$ microscales purchased from Amersham Biosciences (catalog \# RPA 504; RPA 511).

\section{MEASUREMENT OF Arc mRNA EXPRESSION}

Autoradiograms were placed on a Northern Light C-60 illuminator (Research Imaging Inc., Canada) and 12-bit images were acquired with a Hamamatsu ORCA-ER digital camera (Japan) and the Zeiss AxioVision- 3.1 program (Zeiss, Germany). For each triad, autoradiograms of brain sections treated in the same in situ hybridization experiment and originated from WMT, SW and naïve controls were anatomically matched. Quantification of Arc mRNA signals was performed using the ImageGauge-3.36 software (Fuji Film, Japan) by measuring the signal averaged across all layers in the cingulate cortex 1 and $2(\mathrm{Cg} 1 ; \mathrm{Cg} 2)$, retrosplenial (RS), insular (Ins) cortices, medial orbital (MO), ventral orbital (VO), dorso-lateral orbital (DLO) cortices. In addition to Arc measurement averaged over all layers, individual cortical layers were traced in the parietal association (Par), visual primary (V1) and secondary medial and lateral (V2M;V2L) cortices, somatosensory and motor primary and secondary cortices (S1, S2, M1, M2, respectively), frontal association (FrAs), prelimbic (Prl) and lateral orbital (LO) cortices, and lateral entorhinal area (LEA). The boundaries of entire cortical areas and individual layers were carefully verified according to rat brain atlases at multiple rostro-caudal levels (Paxinos and Watson, 1998; Swanson, 2004). The boundaries of cortical layers 2-4, 5 and 6 in Arc mRNA autoradiograms could be clearly determined based on differences in optical densities. The boundaries were confirmed by overlaying the atlas templates over the autoradiograms in the Adobe Illustrator 9.0 program (Adobe Systems Inc; see a tracing example in Figure 4E). To distinguish layers $2 / 3$ and 4 , we relied on the relative thickness of layers $2 / 3$ and layer 4 as shown in a rat brain atlas (Swanson, 2004). We also followed the changes in the layers' width along the rostro-caudal axis. Layer 1 was not labeled and was not traced in this study. Measured mean gray levels were converted into $\mathrm{nCi} / \mathrm{g}$ of tissue equivalents using ${ }^{14} \mathrm{C}$ microscales (Miller, 1991). Polynomial functions of 3-5 orders were used to create calibration curves in Origin 6.1 software (OriginLab Corporation, Northampton, MA, USA).

The radioactive in situ hybridization using ${ }^{35} \mathrm{~S}$-labeled probe is very sensitive in evaluating the overall amount of expressed mRNA and is well suited for precise quantification of neuronal activity (Montag-Sallaz and Montag, 2003). This approach has been 
frequently used in mapping of circuitries underlying learning and memory (Kelly and Deadwyler, 2002, 2003), and developmental plasticity (Tagawa et al., 2005).

\section{PARAMETERS OF Arc mRNA TASK-SPECIFIC EXPRESSION}

In line with the studies that map IEG expression (Zhang et al., 2005), we use Arc mRNA induction as a marker of neuronal genomic activation that occurs during memory tests. This genomic activation may not directly reflect the number of spikes fired by neurons but still indicates brain regions activated by the memory tests. With this consideration, we will further refer to genomic activation of neurons as neuronal activity.

The goal of our study was, first, to establish if there is a relative difference in Arc expression between the trained and two control groups within the two retention groups in multiple cortical areas, and, second, to assess time-dependent changes in task-specific Arc expression. Only this relativistic approach allows dissection of specific effects of training and memory retention length on Arc expression. To overcome possible consequences of the sparse information representation in the cortex (Laughlin and Sejnowski, 2003) that could lead to subtle and sparsely distributed task-related changes in neuronal activity, we applied two types of analyses that capture complementary aspects of activity reorganization.

To elucidate changes in the levels of neuronal activity underlying WM memory recall, we monitored the magnitude of a taskspecific Arc mRNA signal. To be able to use the data from multiple in situ hybridization experiments, we applied a between-subject approach to normalization of Arc mRNA expression levels within the yoked triads. After an in situ experiment, an exact anatomical matching of the sections within a triad was performed. Some sections could not be anatomically matched and were excluded from further analysis. In each triad of anatomically matched WMT, SW and naïve sections treated in the same in situ hybridization experiment, the $\operatorname{Arc} \mathrm{mRNA}$ signal was measured in $\mathrm{nCi} / \mathrm{g}$ of tissue equivalents. For each region of interest (ROI) on each side of a brain section that is a part of an anatomical match, we calculated individual normalized values of Arc mRNA expression. We divided an Arc signal in an ROI in a naïve, WMT or SW brain at $i$-level (naïve, $\mathrm{WMT}_{\mathrm{i}}$ and $\mathrm{SW}_{\mathrm{i}}$, respectively) by the sum of values in this ROI on the same sides of all three matched sections: Naive $_{i, \text { norm }}$ ' naïve $_{\mathrm{i}}+\mathrm{WMT}_{\mathrm{i}}+\mathrm{SW}_{\mathrm{i}} ; \mathrm{WMT}_{\mathrm{i} \text {, norm }}$ naïve $_{\mathrm{i}}+\mathrm{WMT}_{\mathrm{i}}+\mathrm{SW}_{\mathrm{i}} ; \mathrm{SW}_{\mathrm{i}, \text { norm }}$ ' naïve $_{i}+\mathrm{WMT}_{\mathrm{i}}+\mathrm{SW}_{\mathrm{i}}$.

These individual normalized values obtained in multiple in situ hybridization experiments at different rostro-caudal levels of each triad (spanning over 1-8 $\mathrm{mm}$ of the brain) were pooled for scatter plots (maps) of Arc signal distribution against the interaural coordinates for each studied cortical area. Thus, each scatter plot represents the normalized data from WMT, SW and naïve animals from five triads. Finally, the relative magnitudes of learning-specific signals were calculated as the difference between normalized Arc mRNA levels in WMT and SW sections also in each individual anatomical match of each yoked triad.

The numbers of data points per anatomical area/segment varied according to their rostro-caudal extent. Different triads were represented by similar numbers of anatomically matched brain sections. An estimated representation of each animal/triad in the general pool of data points for a given area/segment/layer can be calculated as total number of data points for this area/segment/ layer dived by 5 , the standard error is within $5-8 \%$ of the average sample size.

We performed all statistical analyses on pooled non-averaged data. However, to avoid obscuring of an overall task-specific signal by possible bidirectional changes in the different parts of some extended anatomical areas, we subdivided the areas on segments. Based on a visual inspection of the maps of Arc mRNA signal (scatter plots with pooled data from all the brains), we established the boundaries for cortical segments with the most distinct taskspecific changes or without such changes in recent or remote memory groups. We further applied these common boundaries for all experimental and retention groups (see Tables S1,S2 in Supplementary Material) for a given area/layer. This standard approach to parcellation helps to reveal task-related cortical activity and helps to follow its spatial and temporal dynamics without inducing false positive results (type one errors).

To study a finer functional reorganization in the cortex at the layer level upon memory recall, we applied a within-subject approach to normalization of Arc mRNA laminar expression. To calculate Arc mRNA laminar fractions for a given brain section, absolute values of $\operatorname{Arc}$ mRNA in each layer (Li) were normalized to the sums of Arc mRNA amounts in all layers: $\mathrm{Li} / \mathrm{L} 6+\mathrm{L} 5+\mathrm{L} 4+\mathrm{L} 2 / 3, \mathrm{Li} /$ $\mathrm{L} 6+\mathrm{L} 5+\mathrm{L} 2 / 3$ in the selected 6- or 5-layered areas in WMT and SW groups. The within-subject normalization helps to measure the impact of memory recall on the distribution of the induced amount of Arc mRNA among the different layers. Thus, normalized in this way, changes in the Arc signal estimate changes in the laminar structure of genomic activation of excitatory neurons. This parameter measures excitation flow in the cortical layers on a slower time scale inherent in molecular markers of activity and plasticity (compared to electrical processes).

The interaural coordinates for long-range stereotyped Arc laminar redistributions were identified by visual inspection of the rostro-caudal scatter plots (maps) for Arc mRNA laminar fractions in a given cortical area during WM memory recall and control swimming. Thus, the calculation of these parameters was not limited to individual triads and was determined at the level of the two retention groups.

An experimenter who was not aware of the behavioral conditions traced the cortical layers in a subset of autoradiograms. This blind analysis yielded results that were similar to the outcome of non-blind analysis of the same autoradiograms. To further avoid bias, the data were statistically analyzed only after all the samples were complete. Therefore, the Arc mRNA signal measurements were taken without benefit of advance knowledge of effects that emerged.

\section{STATISTICAL ANALYSES}

Normality of data distributions and variance equality were verified by the Kolmogorov-Smirnov test and $F$-test, respectively. The acquisition of spatial memory in the Morris water-maze task was evaluated by repeated measures ANOVA. Performance on recent and long-term spatial memory tasks was assessed by one-way ANOVA followed by a Fisher's post hoc test and a Kruskal-Wallis test where appropriate on individual probe tests (Statview; SAS Institute, Cary, NC, USA). The presence of a learning-specific signal in the individual 
segments of the scatter plots for each cortical area was assessed by region-by-region univariate ANOVAs followed by a Fisher's post hoc test on pooled data as described early (Gusev et al., 2005). The main and combined effects of a memory retention period, a cortical segment and a layer's position on learning-specific Arc mRNA expression were evaluated by two- and three-way ANOVAs on pooled data followed by Scheffe's post hoc test. Scheffe's test was applied as it is robust to differences in sample sizes across different segments. Effects of the time elapsed since memory acquisition on magnitudes of task-specific signal were assessed by Student's $t$-tests only in pairs of analogous cortical segments, no cross comparison was performed by $t$-tests. Effects of a behavioral protocol on Arc mRNA laminar fractions were assessed by Student's $t$-tests in a different set of cortical segments. Single comparisons of analogous segments by the $t$-tests do not require Bonferroni adjustments. Null hypotheses were rejected at $p<0.05$.

\section{RESULTS \\ SPATIAL WATER-MAZE MEMORY DOES NOT DECLINE OVER 1 MONTH AFTER TRAINING}

A persistent issue in remote memory research is how to distinguish memory modifications or even deterioration due to the age of the memory from modifications resulting from memory inaccessibility due to difficulties in remote recall (Biedenkapp and Rudy, 2007; Wiltgen and Silva, 2007). This uncertainty prevents firm conclusions about time-dependent changes in behavioral content and neuronal circuits during memory consolidation and its permanent storage. We introduced a methodology that ameliorates the storage/recall uncertainty in our previous hippocampal study (Gusev et al., 2005). The brains from these same rats are used in the current study. We now map neocortical regions involved in systems-level consolidation of a spatial memory task.

To further validate our training protocol and task performance analysis, we present newly obtained data on reminding (for details, see Figure S2 in Supplementary Material) and on proximity to the platform location.

In brief, groups of animals trained for studying recent and remote spatial memories displayed a similar time course of WM learning (Figures 1A,E). Probe tests indicated a clear spatial preference for the target location both $24 \mathrm{~h}$ and 1 month after the last training session (Figures 1B,F, respectively). There were higher numbers of crossings for target quadrant centers (hidden platform location) compared to non-target quadrants recorded for each trained animal during three consecutive probe tests. On the other hand, swim yoked control rats for both the 24-h and 1-month retention groups did not demonstrate spatial bias as measured by numbers of crossings of "target" quadrant centers compared to non-target quadrants also recorded for each animal during three consecutive "probe" tests Figures 1C,G). There was no memory decline over a 1-month retention interval measured by a number of parameters (Gusev et al., 2005). The quadrant analyses of target/non-target quadrant center crossings did not indicate difference between the 1 -month probe tests as compared to 24 -h tests (Figures 1B,F).

The three consecutive probe tests allowed us to monitor recall dynamics. Neither the recent nor the remote memory group indicated search behavior extinction in any parameter. We observed a robust improvement in the platform search in two out of five
WMT animals in the remote memory group. These two animals showed a shorter latency to platform area crossing, a closer average proximity to the platform area and an increased dwell time/distance in the target quadrant during the second and third probe tests compared to the first test. The most significant improvement occurred after the first probe whereas performance in the second and third probes was similar. As we reported previously, this tendency toward performance improvement, however, did not reach overall statistical significance for the entire remote memory group (Gusev et al., 2005). Also, average proximity to the platform area did not change significantly in either the recent or remote memory groups over the three tests $(p>0.24, p>0.41$, respectively; Kruskal-Wallis test). Thus, extended exposure to the training room context followed by the three consecutive probe tests ameliorated possible recall difficulties at the recent and especially the remote time points.

To further validate our training protocol for induction of a robust remote memory, we measured a proximity parameter. A two-way ANOVA indicated significant main effects of "memory retention time" and "behavior" factors on the proximity of rats to the platform location $\left[F_{(1,50)}=27.57, p<0.0001 ; F_{(1,50)}=42.81, p<0.0001\right.$, respectively], but the factors' interaction was not significant $\left[F_{(1,50)}=0.002\right.$, $p=0.96$; Figures $1 \mathrm{D}, \mathbf{H}]$. The WMT rats swam in closer proximity to the platform location when compared with SW control rats, and this difference did not change over 1 month. One-way ANOVAs with "animal group" as a factor indicated the differences in WMT and SW proximities within both the 24-h and 1-month memory groups $\left[F_{(3,50)}=25.31, p<0.0001 ; p<0.0001, p<0.0001\right.$, Fisher's post hoc test, respectively]. There was also an overall decrease in proximity to the platform at 1 month compared to 1 day for both WMT and SW rats $(p<0.0001)$. However, the interaction of the main factors including "memory retention time" and "behavior" was not significant. This indicates that, by the proximity measure, WM memory also did not decline over a 1-month retention interval. In addition, quadrant dwell times and distances also indicated robust remote memory (Figure S1 in Supplementary Material).

Differences in total distance swum and/or corticosterone levels may affect $A r c$ mRNA expression. There was, however, no difference in the total length of swim pathways during the memory probe tests and control swim within both retention groups (Figures 1I,J). There were also no differences in corticosterone levels in WMT and SW rats after either training room exposure (Figure 1K) or the final sessions of probe and control tests (Figure 1L).

Thus our behavioral protocol not only induced robust memory but also created conditions that ameliorated difficulties with remote recall. The behavioral controls for non-spatial and stress components allowed us to extract memory-related neuronal activity and its modification over time. To elucidate time-dependent changes in putative storage sites for WM memory, we examined regional (spatial) selectivity in neocortical activation during recent and remote memory recall.

\section{TASK-SPECIFIC INCREASE IN OVERALL ArC GENE EXPRESSION OCCURS IN SELECT ROSTRO-CAUDAL SEGMENTS. THE SPATIAL DISTRIBUTION OF THE Arc SIGNAL DIFFERS AFTER RECENT AND REMOTE RECALL}

We first analyzed overall Arc expression levels averaged across cortical layers. A detailed laminar analysis for most of areas was conducted as well. With the first approach, we deciphered spatial 


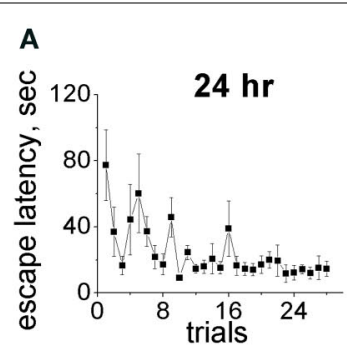

E

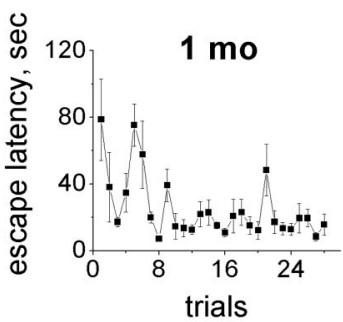

I

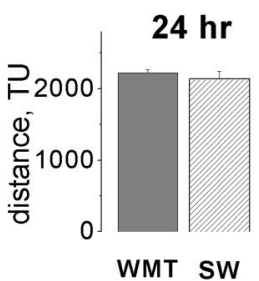

B

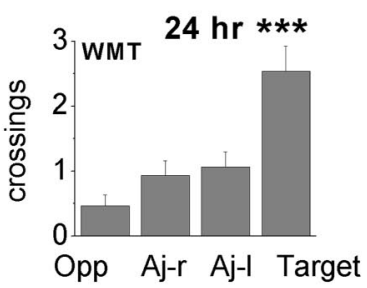

$\mathbf{F}$

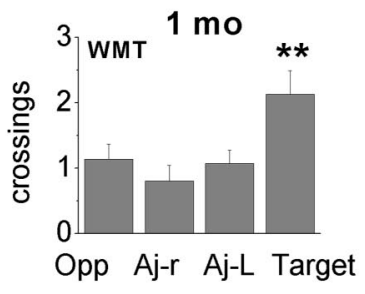

$\mathbf{J}$

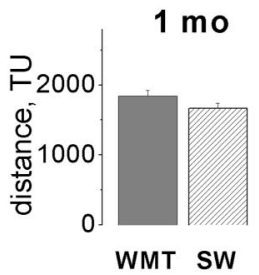

C

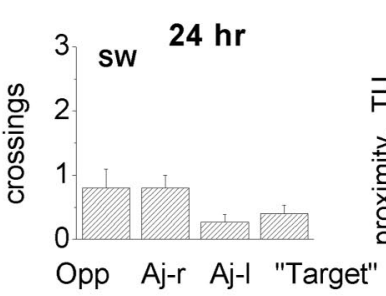

G
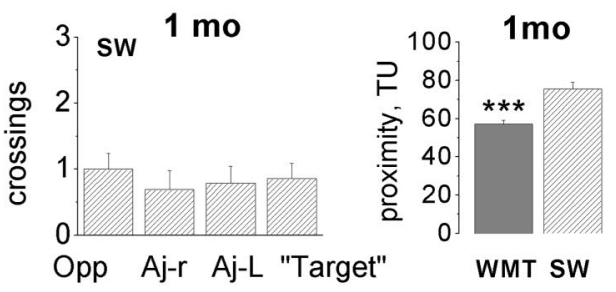

K

$\mathbf{L}$

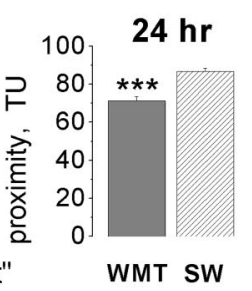

H

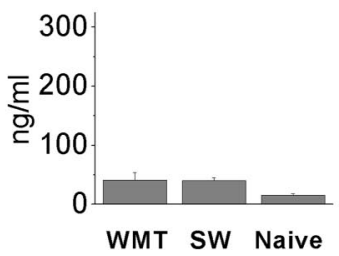

FIGURE 1 |Acquisition and long-term retention of spatial memory water-maze task. $(\mathbf{A}, \mathbf{E})$ Escape latency dynamics during WM training. $(\mathbf{B}, \mathbf{F})$ Probe tests performed $24 \mathrm{~h}(\mathbf{B})$ and 1 month $(\mathbf{F})$ after 7 days of water-maze (WM) training. Bars display means \pm SE of number of crossings over the target location and non-target quadrant centers. Altogether, 15 tests were conducted in five triads of rats. Note the spatial bias in the swim paths of water-maze trained (WMT) rats. Target quadrant contained an escape platform during training trials, Opp-quadrant, opposite to target quadrant, Aj-r, adjacent right; Aj-l, adjacent left quadrants. (C, G) There was no spatial bias in the swim paths of swim yoked controls (SW) in the tests conducted $24 \mathrm{~h}$ and 1 month after the last training session. $\mathbf{( D , H )}$ Average proximity to the platform location during recent
(D) and remote (H) memory tests. WM trained rats persistently swam closer to the target location compared to swim controls. $(\mathbf{I}, \mathbf{J})$ There was no difference in the distance swum by WMT and SW rats at $24 \mathrm{~h}$ (I) or 1 month (J) after training (during the final sessions before the Arc mapping). (K,L) There was no difference in corticosterone levels in WMT and SW rats both after training room exposures (K) and after the final sessions of probe and control tests (L). Data represent means $\pm \mathrm{SE},{ }^{* *} p<0.02,{ }^{* *} p<0.0001$ one-way ANOVA with Fisher's post hoc test. For simplicity, statistically significant differences indicated between target and all non-target quadrants, and between WMT, SW and naïve groups only within memory retention groups. Adapted from Gusev et al. (2005); TUtracker units. selectivity in global cortical activation by a memory recall. We showed that appearance of task-specific Arc signal depends on cortical segment coordinates. This selectivity in task-specific Arc expression became more pronounced at the 1-month retention. Fewer regions were activated at the remote vs. recent time point.

To estimate activity volume of putative excitatory neurons, we used between-subject normalization of Arc mRNA amounts within the individual animal triads. We surveyed 40 maps for 20 entire anatomical areas representing Arc mRNA levels plotted against the interaural brain coordinates for recent and remote recall. The initial survey indicated that the direction of task-specific changes in Arc signal was not always similar at different rostro-caudal levels within some of the cortical areas. Therefore, to avoid obscuring memory-related activity, 17 areas were subdivided into rostrocaudal segments that displayed similar directions in Arc alterations after memory recall. We used the same boundaries of 43 individual segments among all experimental and retention groups and then studied spatial and time-dependent changes in the Arc task-specific signal among these cortical segments.

We first analyzed the directionality of Arc expression changes and spatial distribution of such changes across the entire cortex. The differences between the normalized Arc mRNA values averaged across all layers in WMT and SW rats revealed task-specific signals (Figure 2).

Following recall of both recent and remote memories, the most frequent task-specific signal we detected was an increase in Arc expression. We used multiple one-way ANOVAs followed by Fisher's post hoc test to asses significance of the task-specific difference in individual segments (for the boundaries of the studied cortical segments and the results of statistical analyses, see Tables S1 A,B and S2 in Supplementary Material). Remarkably, the numbers of segments showing increased Arc expression declined from 30 (69.8\%) to 

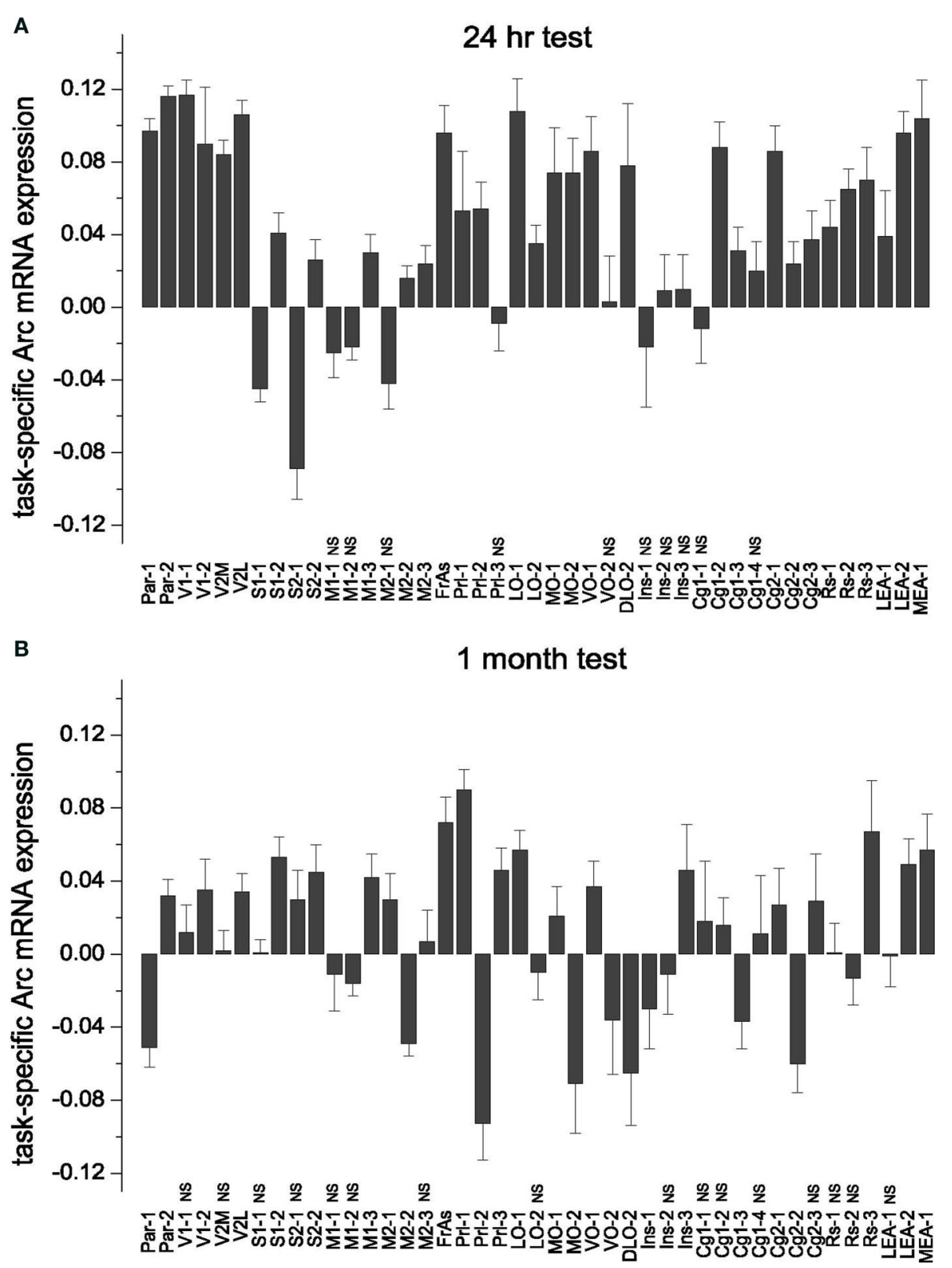

FIGURE 2 |Task-specific changes in overall (averaged across the all layers) Arc gene expression: subdivision of cortical areas, stereotypical magnitude of $A r c$ expression increase and its area-specific persistence over 1-month memory consolidation. (A,B) Both during recent and remote memory activation, the post hoc analyses distinguished only cortical segments that had opposite changes in Arc expression, or the segments with and without changes in Arc expression. The latter segments had statistically non-significant (NS) differences in Arc expression levels and indicated with NS. There was no difference in magnitude of learning-specific signals within the groups of cortical segments with more or less Arc gene expression compared to swimming controls. (A) There was no task-specific signal in M1-1, M1-2 and M2-1, Prl-3, VO-2, Ins-1, Ins-2 and Ins-3, Cg1-1, Cg1-4 (Table S1A in Supplementary

$18(41.9 \%)$ over a 1-month period. Numbers of segments showing less Arc expression or no task-specific Arc alterations increased from $3(7 \%)$ to $9(20.9 \%)$ and from $10(23.3 \%)$ to $16(37.2 \%)$, respectively (Figure 2 ).
Material). MEA and LEA data are taken from our previous publication (Gusev et al., 2005) and included here for the purpose of completeness. Data represent group means \pm SE for differences in Arc mRNA levels between WMT and SW. (B) The number of segments with less Arc mRNA expression was higher after remote recall compared to resent recall. A new decrease in Arc expression appeared in VO-2 and Ins, a new increase in Arc expression appeared in S1-1, S2-1, Prl-1, Ins-3, M2-1, M1-3 segments (Table S1B in Supplementary Material). When Arc expression levels were averaged across the layers, there was no learning-specific signal detected in V1-1, V2M, M1-1, M2-3, LO-2, RS-1, RS-2, Cg1-1, Cg1-2, Cg1-4, Cg2-3, Ins-2 segments (all $p>0.05)$. V1-2, S1-1 and S2-1 displayed learning-specific signal only in layers $2 / 3$ during remote recall.

Stronger task-specific activity in a region may indicate its stronger relation to memory storage. Therefore, we next examined how the magnitude of task-specific Arc expression varied among cortical areas and whether this magnitude differed after recent 
and remote memory recall. Two-way ANOVA indicated significant main effects of "cortical segment" and "memory retention time" factors on the magnitude of Arc mRNA task-specific expression [as averaged across all layers, $F_{(42,9598)}=13.79, p<0.0001$; $F_{(1,9598)}=67.62, p<0.0001$, respectively], and the factors' interaction was also significant $\left[F_{(42,9598)}=8.44, p<0.0001\right]$. Thus, appearance of task-specific Arc signal depends on both cortical segment coordinates (spatial selectivity) and the retention time. Moreover, individual segments even within the same cortical areas may differ in how direction and magnitude of the Arc task-specific signal changes over 1 month.

To examine whether memory recall/storage functions are similarly represented across participating cortical areas of different sensory modalities and hierarchy, we next tested the hypotheses that task-specific activity has a stereotypical overall magnitude and similar laminar distribution across multiple cortical segments. We also examined whether character of stereotypy in cortical activation differed after recent and remote memory recall.

\section{THE MAGNITUDE OF INCREASE IN Arc mRNA EXPRESSION IS STEREOTYPICAL ACROSS THE ACTIVATED SEGMENTS}

The term "stereotypy" describes the changes in Arc mRNA expression that are similar in their magnitude and/or laminar distribution following memory recall. Such changes repeat across the cortical areas of different modalities that express Arc levels specific to WM memory recall.

To test the hypothesis that memory recall is accompanied by a stereotypical increase in cortical activity, we compared the magnitude of task-specific Arc signals across the segments within the 24-h and 1-month memory groups. We applied one-way ANOVAs with cortical segments as a factor followed by Scheffe's post hoc analyses. After both recent and remote memory activation, the post hoc analyses distinguished only cortical segments in which Arc expression changed in opposite directions, and the segments with and without statistically significant alterations in Arc levels. There was no difference in magnitude of $A r c$ task-specific signals within the groups of cortical segments that changed in the same direction. Thus the magnitude of Arc change in these segments was stereotypical (Figure 2).

Specifically, after the recent memory recall, the task-specific Arc signals in the Par-1, Par-2, V1-1, V2M, V2L, FrAs, LO-1, LEA-2 and VO-1 segments differed from the Arc signals in S1-1, S2-1, M1-2, M2-2, M1-1, M2-1 segments $\left[F_{(42,4517)}=18.64, p<0.0001\right.$; Scheffe's post hoc, all $p<0.05]$. The first group of segments displayed more Arc mRNA expression after memory recall when compared to swim controls, and the second group had less Arc mRNA expression in all or only selected layers (Figure 2A; also see Table S1A in Supplementary Material). There was, however, no statistically significant difference in magnitude of Arc task-specific expression within the first group of segments with more Arc mRNA (Par-1, Par-2, V1-1, V2M, V2L, FrAs, LO-1, LEA-2 and VO-1); nor was there a difference in magnitudes of task-related Arc expression between the first group of segments and another group with more Arc expression including S2-2, Prl-2, LO-2, MO-1, MO-2, DLO-2, Cg1-2, Cg1-3, Cg2-1, Cg2-2, Cg2-3, RS-1, RS-2, RS-3, MEA-1, LEA-1 segments $(p>0.05$; Figure $2 \mathrm{~A})$.
After the remote memory recall, similar to the recent recall, Scheffe's post hoc analysis indicated no difference in relative magnitudes of task-specific signal among the segments that displayed more Arc mRNA expression (Par-2, V1-2, V2L, S1-2, S2-2, M2-1, M1-3, FrAs, Prl-1, Prl-3, LO-1, MO-1, VO-1, Ins-3, RS-3, LEA2, MEA-1 segments; $F_{(42,5081)}=6.63, p<0.0001$, but all corresponding $p>0.05$; Table S1B in Supplementary Material; Figure 2B). However, the magnitude of Arc task-specific expression in FrAs and Prl-1 differed from the magnitude of Arc task-specific expression in Par-1 $\left[F_{(42,5081)}=6.63, p<0.0001 ; p<0.03, p<0.02\right]$ and M2-2 $(p<0.03, p<0.02)$ which displayed less Arc mRNA expression.

Thus, after performance on a WM memory task, more or less Arc expression occurs only in select rostro-caudal segments when compared to controls. This spatial restriction in activation suggests a functional subdivision of cortical areas in providing WM memory recall. However, the overall magnitudes of Arc gene expression induced by memory recall remain similar (stereotypical) across the multiple activated segments within each retention group. The spatial restriction in $\operatorname{Arc}$ increase is more pronounced after a 1-month memory recall when fewer segments show Arc increase compared to recent recall.

\section{PERSISTENT REPRESENTATION OF MEMORY RECALL: ONLY SELECT SEGMENTS DISPLAY A SIMILAR INCREASE IN Arc mRNA EXPRESSION AFTER BOTH RECENT AND REMOTE RECALL}

Stronger Arc expression may indicate more neurons and/or more pronounced $A r c$-related mnemonic plasticity representing early or remote memory storage. Therefore, after revealing time-dependent changes in the spatial distribution of Arc cortical signal, we examined whether Arc signal magnitude also differs after recent and remote memory recall.

We found an overall significant decrease in the magnitude of task-specific Arc signals in the cortex induced by recall of the remote WM task when compared to the recent task $(0.044 \pm 0.002$ and $0.015 \pm 0.002 ; F_{(1,9682)}=85.78, p<0.0001 ; p<0.0001$, Scheffe's post hoc). Moreover, the statistically significant interaction of "cortical segment" and "memory retention time" factors in two-way ANOVA (see above) indicated that the directionality and/or magnitude of task-specific Arc signals over a 1-month retention interval is area-specific. We therefore assessed the changes in task-specific Arc expression in pairs of analogous individual segments by unpaired t-tests (at $p<0.05$ ) over a 1 -month period.

We found that the magnitudes of Arc task-specific expression in FrAs, Prl-1, MO-1 (for representative images see Figure 3), S1-2, S2-2, M1-3 and RS-3 segments were similar after both recent and remote recall ( $p>0.05$; Figure 2; for all large-scale maps of Arc expression averaged across all layers see Figure S4 in Supplementary Material). We also found that the Arc task-specific expression of smaller magnitude persisted in Par-2, V1-2, V2L (for representative images see Figure 5 and Figure $\mathbf{6}$ in Supplementary Material, respectively), in LO- 1 and VO- $1, \mathrm{Cg} 2-1$ segments $(p<0.05)$. The direction in task-specific Arc expression had inverted in Par-1, M2-2, PrL-2, DLO-2, MO-2, Cg1-3, Cg2-2 segments where Arc signal magnitude diminished in WMT compared to SW control $(p<0.05)$. The magnitude of task-specific Arc expression increased in WMT in M2-1, PrL-3 and Ins-3 segments $(p<0.05)$. Finally, segments such as DLO-1, Ins-3, and M2-1 displayed more Arc expression only after remote recall. 


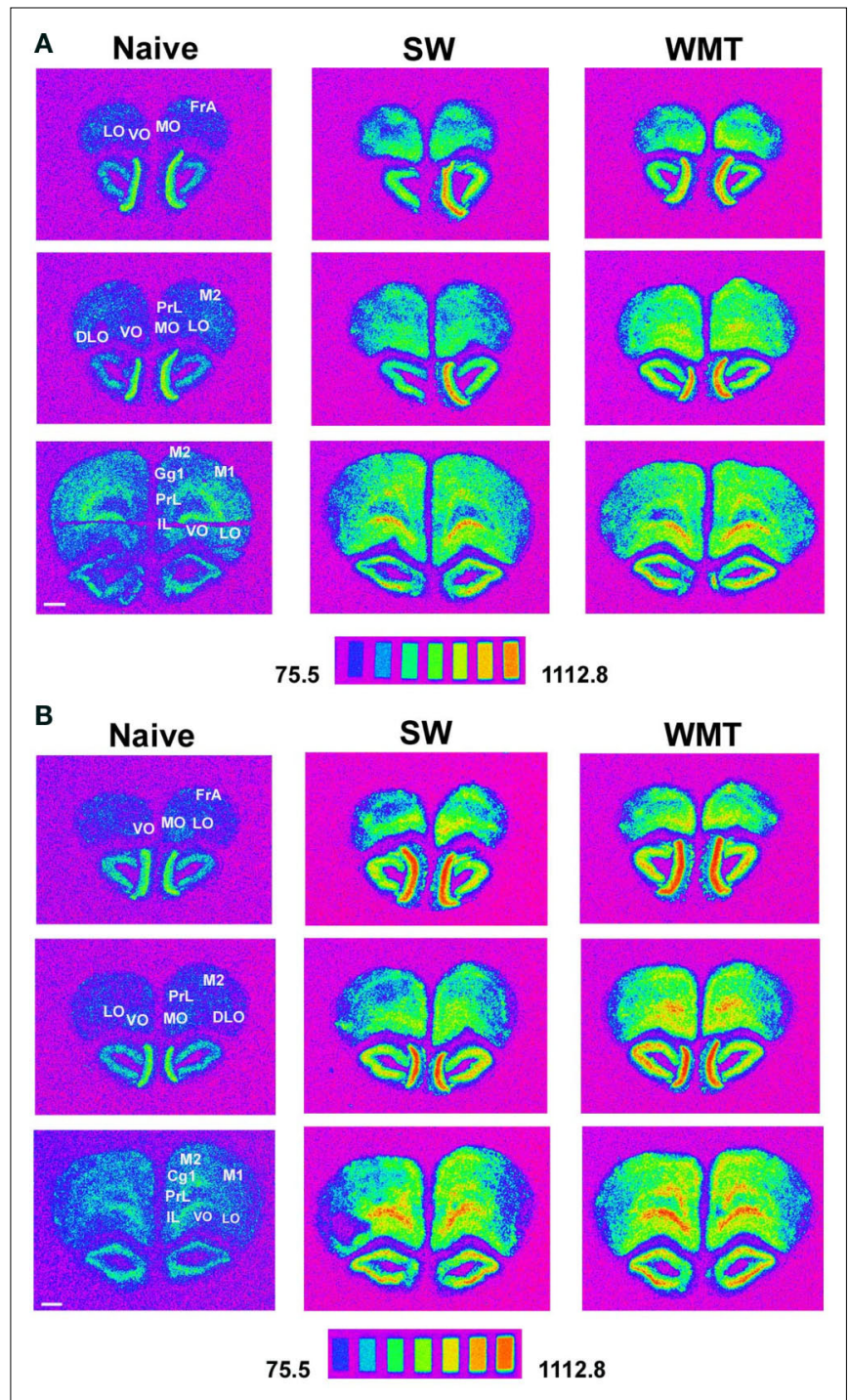

FIGURE 3 |Areas of the prefrontal cortex expressed more Arc mRNA than controls after spatial memory recall at 24-h and 1-month retention intervals. (A) Spectrum images of autoradiograms show more Arc mRNA expression in FrAs, Prl, Cg1, M1, M2, MO, LO following a recent memory test in a WMT rat compared to its SW and naïve anatomically matched control sections. Images of the ${ }^{14} \mathrm{C}$ microscales that were exposed in parallel with the samples illustrate the conversion of a mean gray level into $\mathrm{nCi} / \mathrm{g}$ of tissue equivalents. Scale bar: $1 \mathrm{~mm}$. (B) Autoradiograms show characteristic for long-term memory recall more Arc mRNA expression in FrAs, Prl, MO, LO, $\mathrm{M} 1$ and M2 of a WMT rat compared to its SW and naïve controls. Note that displayed images represent different in situ hybridization experiments and therefore cannot be compared directly over the memory retention period. Experimental triads 4 and 10 represent recent and remote recall, respectively.

Thus, the evolution of Arc signal magnitude over a 1-month period is area-specific. The changes in Arc increase together with Arc expression clustering suggest that cortical areas may differ in the persistence of their roles in memory recall and/or storage.

Detection of enduring neuronal correlates for memory storage is impeded by sparse information representation in the brain. This difficulty, however, could be partly ameliorated if laminar distribution of storage among cortical sites follows a stereotypical organization.
Such a distribution would point to storage locations that are common across the entire cortex. We therefore constructed detailed maps of cortical laminar activity underlying memory recall.

\section{RECENT BUT NOT REMOTE RECALL INDUCES MORE Arc EXPRESSION IN THE UPPER LAYERS OF PARIETAL AND VISUAL CORTICES}

A distinct repeating motif in the laminar profiles of task-specific Arc gene expression appeared across several regions only following recall of recent but not remote memory (Figure 4). The magnitude of Arc expression specific to learning tended to be larger in the uppermost layers (2-4) after only recent recall. After remote recall, the magnitude of Arc task-specific expression did not systematically differ across the cortical layers (compare Figures 4A,B and Figures 4C,D; for detailed analyses of Arc laminar expression across the multiple segments see Tables S1A,B in Supplementary Material).

Specifically, at the level of the entire population of 6- and 5-layered regions, the task-specific signal in L6 had the smallest magnitude when compared to signals in L5, L4 and L2/3 $\left[F_{(3,3556)}=\right.$ 15.65, $p<0.0001 ; p<0.003, p<0.0001, p<0.0001$, respectively; Scheffe's post hoc; Figure 4F], but the layers did not differ after the 1 -month tests $\left[F_{(3,3877)}=0.74, p=0.53\right.$; Figure 4G]. The Arc signal specific to the recent WM task in L5 was smaller when compared to signal in L4 $(p<0.0092)$; the Arc signal magnitudes in L5 and $\mathrm{L} 2 / 3(p=0.8), \mathrm{L} 4$ and L2/3 ( $p=0.08)$ did not differ.

At the level of individual cortical segments, the laminar differences in magnitude of task-specific Arc expression were significant within the combined Par- 1 and Par- $2\left[F_{(3,430)}=4.45, p<0.005\right]$, and V1-1 and V1-2 $\left[F_{(3,240)}=3.57, p<0.02\right]$ regions. In the parietal association cortex, the strongest task-specific signal appeared in $\mathrm{L} 2 / 3$ and L4 when compared to both L6 $(p<0.006, p<0.003$, Fisher's post $h o c)$ and L5 ( $p=0.055 ; p<0.03$; Figure 4). In V1, the increase in Arc expression was the highest in L2/3 and L4 as compared to L6 only $(p<0.003 ; p<0.03$, Figure S6 in Supplementary Material). In the entire parietal and $\mathrm{V} 1$ regions, $\mathrm{L} 2 / 3$ and L4, L6 and L5 task-related signals did not differ $(p>0.8, p>0.8 ; p>0.4, p>0.4)$.

At 1 month in memory retention, a task-specific signal emerged in all layers of Par-2, FrAs, Prl-1, LO-1 segments, but it appeared only in selected layers of V1-2, V2L, S1-2, S2-1, S2-2, M1-3 and M2-1 regions (Figures 4C,D; Table S1B in Supplementary Material; for representative images and $\operatorname{Arc}$ laminar maps for parietal and visual areas see Figure 5 and Figure S6 in Supplementary Material). We found that a similar percentage of the upper and deep layers (50 and 57\%) demonstrated more Arc expression specific to the task both after the recent and remote recall.

Thus, the task-specific laminar profiles of Arc increase change as memory consolidates. By this measure, the representations of recent and remote memory recall overlap in various layers depending on the cortical area (compare Table S1A and Table S1B in Supplementary Material; for all laminar maps based on between-subject normalization of Arc signal see Figure S3 in Supplementary Material).

\section{MEMORY RECALL INDUCES STEREOTYPICAL REDISTRIBUTION OF ArC LAMINAR EXPRESSION, BUT THE REDISTRIBUTION PATTERNS DIFFER BETWEEN RECENT AND REMOTE RECALL}

To determine reorganization in laminar patterns of excitation distribution underlying WM memory recall, we studied Arc laminar distribution. The Arc laminar fractions were calculated based on 

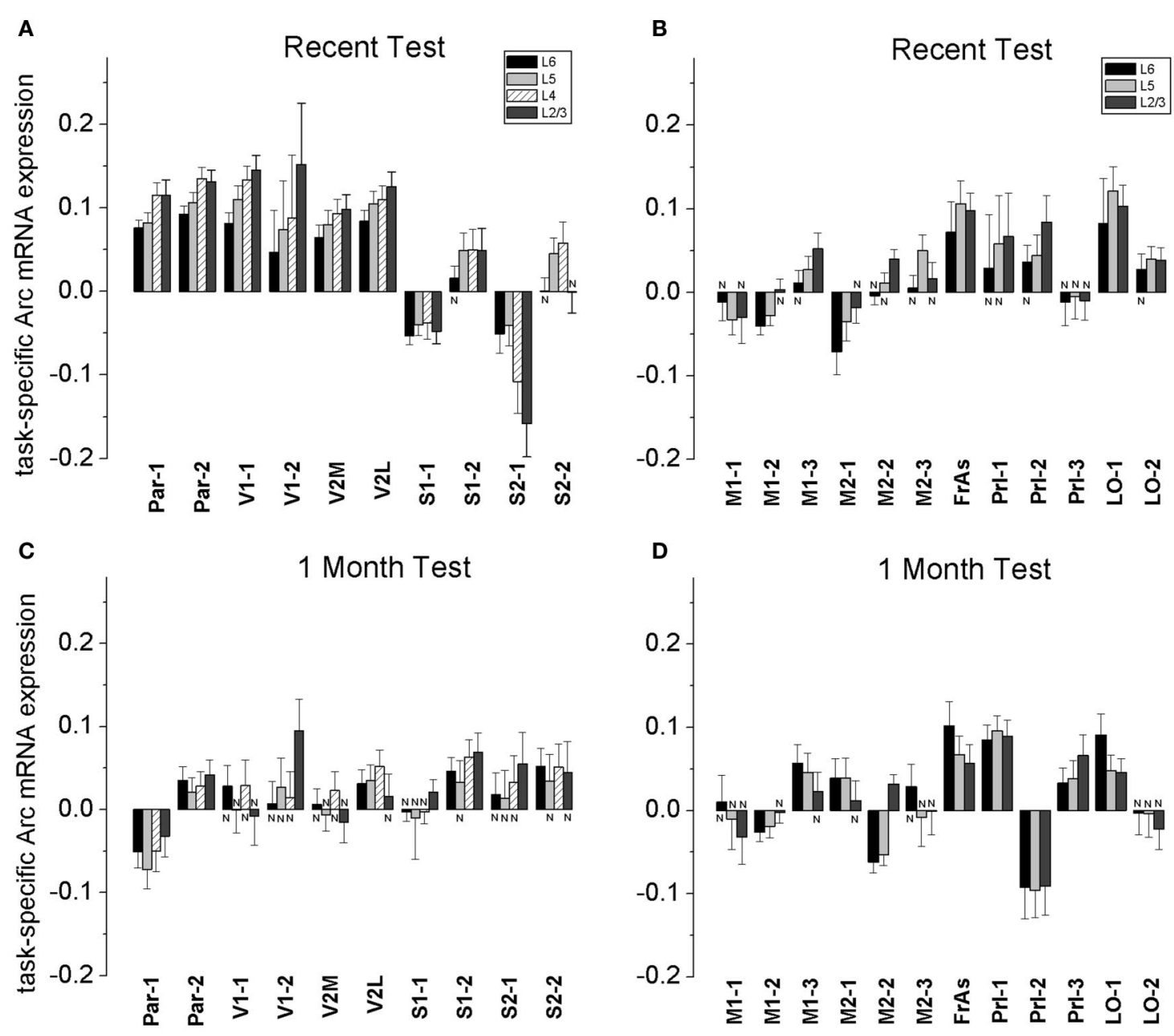

E

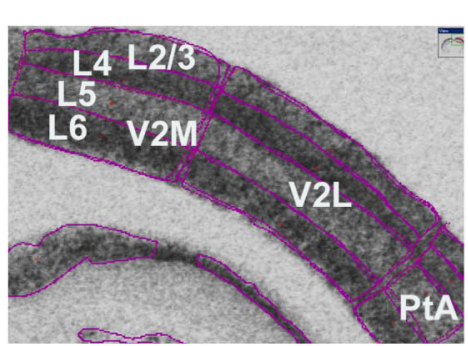

$\mathbf{F}$

$$
\left.\begin{array}{l}
0.10 \\
0.08 \\
0.06 \\
0.04 \\
0.02 \\
0.00
\end{array}\right]
$$

FIGURE 4 | A stereotypical laminar profile of task-specific increase in Arc expression levels appeared after recall of recent but not remote memories. $(\mathbf{A}, \mathbf{B}, \mathbf{F})$ A recent memory test was accompanied by a larger increase in Arc $m R N A$ expression in $L 2 / 3$ and $L 4$, while the increase in Arc expression was less in L6 in Par, V1, V2M and V2L, S1-2 segments. The tendency for the smaller L6 increase in Arc signal was also preserved in 5-layered areas such as FrAs, Prl, LO, M1-3 and M2-2. Data represent group means \pm SE of WMT and SW Arc mRNA differences, N-the WMT vs. SW difference is not significant. (E) The layers' boundaries were traced with confidence. $L 2 / 3$ and $L 4$ boundaries were
G

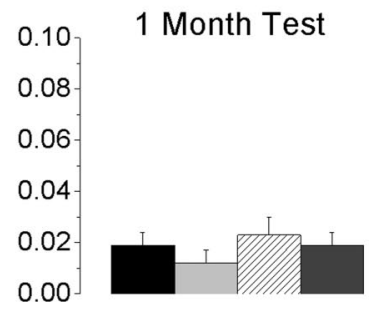

verified with a rat brain atlas. A screen shot from the ImageGauge program is shown. (C,D,G) After a 1-month memory interval, a stereotypical laminar profile for the magnitude of task-specific Arc signal was not found. Instead, a significantly reduced signal was restricted to only some upper and/or deep layers in a fewer regions. Thus, by this measure, overlap in representations of recent and remote memory recall occurred in various layers depending on the cortical area (compare A and C, B and D). The FrAs, Prl-1, LO-1 expressed more Arc mRNA in WMT rats compared to SW rats both after the recent and remote memory recall. within-subject $A r c$ signal normalization. For 11 cortical anatomical areas listed in the Section "Materials and Methods", we constructed and examined 86 plots of task-specific Arc mRNA laminar distribution. We determined the coordinates for rostro-caudal segments with the most distinct changes in the Arc fractions. These common boundaries were applied within the subdivided anatomical areas across the 24-h and 1-month groups during statistical analyses of Arc laminar fractions. 

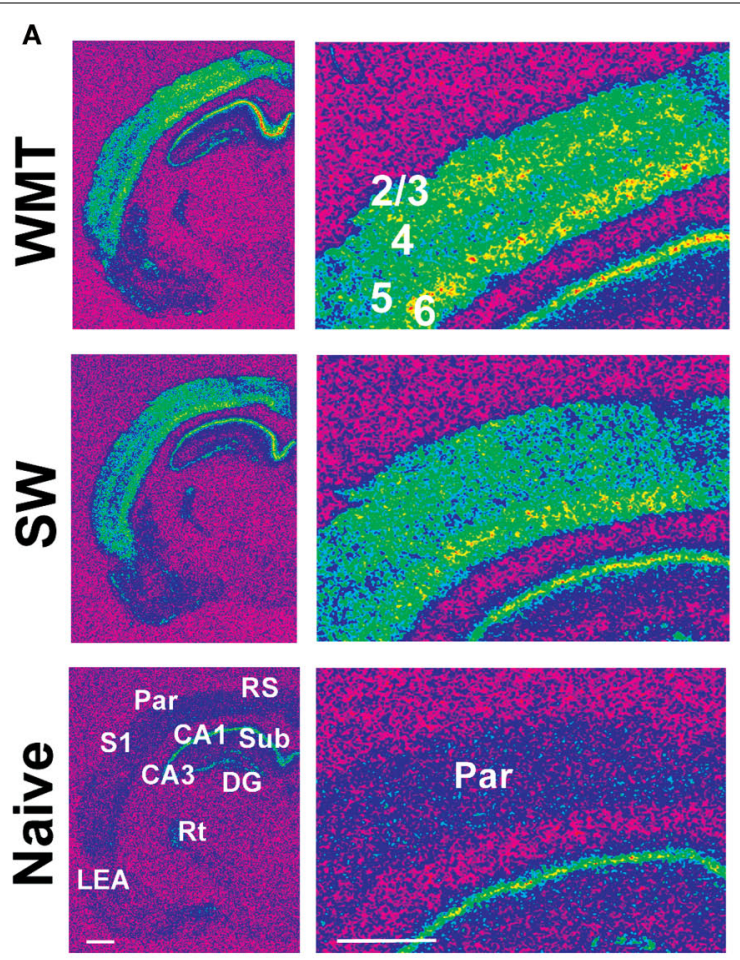

75.5
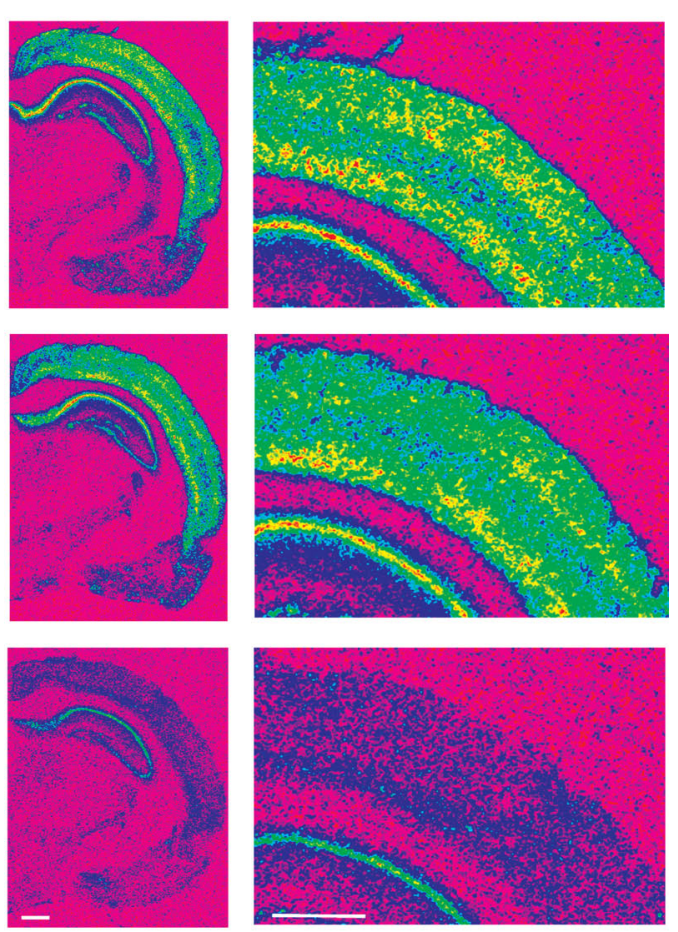

$\mathbf{7 5 . 5}$

1112.8

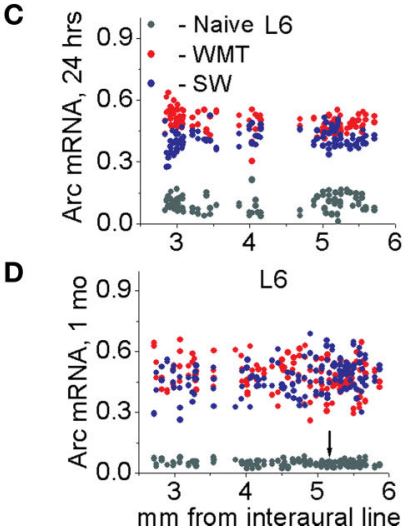

C

$\mathrm{mm}$ from interaural line

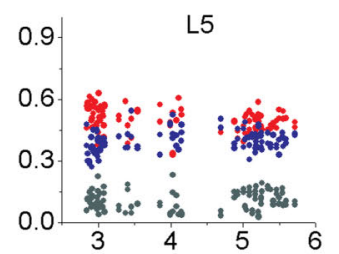

0.9. L5

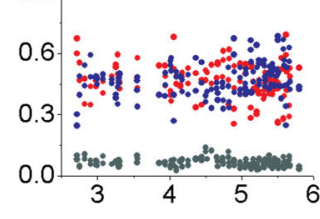

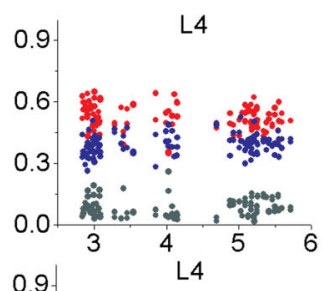

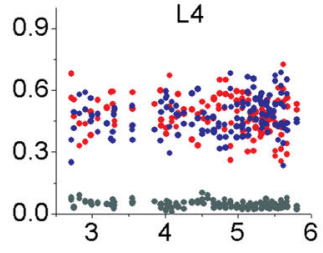

0.9

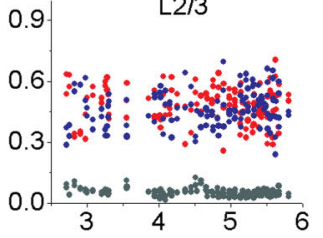

FIGURE 5 | Multiple rostro-caudal levels of the parietal association cortex show time-dependent changes in laminar profiles for Arc gene expression specific to water-maze memory recall. $(\mathbf{A}, \mathbf{C})$ Following a recent memory recall, stronger Arc mRNA expression occurs consistently in the upper layers when compared to the deep layers. The spectrum images of autoradiograms were obtained from brain sections of WMT, SW and naive control rats. For the illustrated sections, a task-specific Arc signal is $304.3 \mathrm{nCi} / \mathrm{g}$ for the deep (L5 and L6) layers and $472.1 \mathrm{nCi} / \mathrm{g}$ for the upper (L2/3 and L4) layers. A task-specific signal is the difference in absolute values of Arc mRNA expression between the WMT and SW sections. The scatter plots show that a stronger Arc expression was found in WMT rats relative to SW and naïve matched controls in all layers at the multiple rostro-caudal levels. The data points in the scatter plots are individual mean values of Arc mRNA levels obtained by between-subject normalizations for anatomical matches pooled from the five triads. (B,D) Following a remote memory recall, a stronger task-specific Arc expression was found in the upper layers compared to the deep layers but only in a few sections located around 5-mm level (100.5 and $275.3 \mathrm{nCi} / \mathrm{g}$, respectively; see images on B). Overall, however, the difference between upper and deep layers diminished and was not consistent at this point. After a 1-month memory recall, the caudal part of the parietal cortex (Par-2, within a 5.19-2.5 mm segment, indicated by an arrow) displayed a smaller increase in Arc expression. In the rostral part (Par-1, within a 5.8-5.2 mm segment), the Arc signal in the WMT group became even smaller than the SW signal. Scale bar: $1 \mathrm{~mm}$. The brain sections in (A,B) were obtained from experimental triads 1 and 9, respectively.
Following performance on a recently learned WM task, a distinct pattern of Arc laminar redistribution repeated within a subset of cortical areas with different modalities and layer arrangements such as Par, V1, V2L and V2M, M1 and M2, and FrAs. Relative to SW controls, Arc
mRNA fractions became larger in the upper layers $\mathrm{L} 4$ and/or L2/3, but they became smaller in L6 and did not change consistently in L5 (for representative stereotypical redistribution maps across the parietal association, primary visual and motor areas, see Figures 6A-D; for 


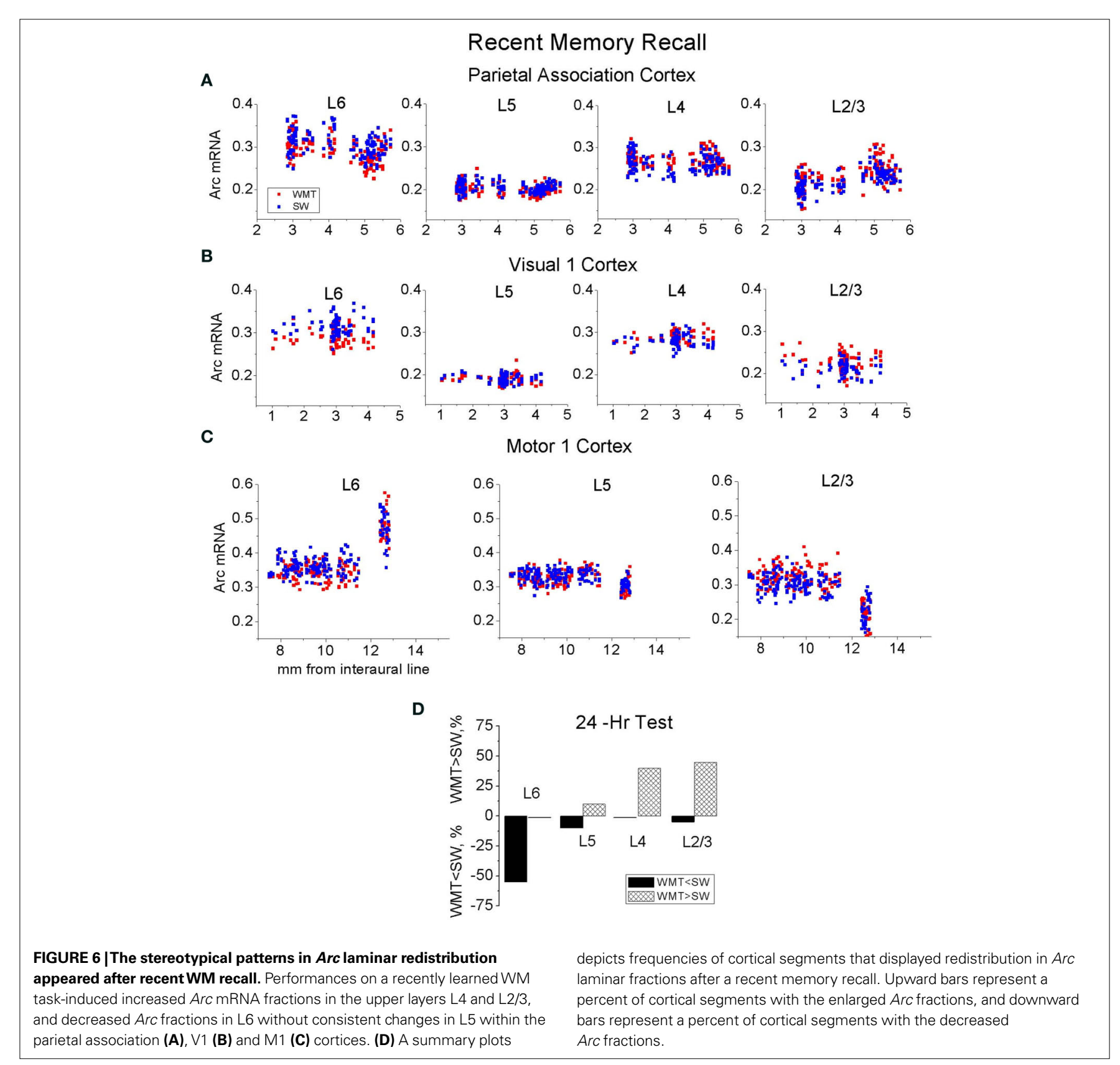

results of the statistical analyses and the boundaries of all segments see Table S3 in Supplementary Material). The S1, S2, Prl and LO areas did not demonstrate this Arc laminar redistribution.

The pattern of bidirectional changes in Arc mRNA distribution was, however, time-dependent. Arc fractions in L6 did not decrease consistently after the 1-month test. The frequency of Arc fractions' increase in the upper layers still maintained (Figure 7). While the Par-1, V1-1, V1-2 and V2M-1 segments had enlarged L2/3 and/ or L4 Arc fractions persistently, the V2L-1, V2M-2, S1, and Prl-2 segments showed such increases in L2/3 and L4 fractions only at 1-month retention. Only M1-2 and M2-2 segments reproduced the complex redistribution pattern in its entirety over a 1-month retention period (for all maps of Arc laminar redistribution see Figure S5 in Supplementary Material).
Thus, performance on WM tasks induces stereotypical Arc laminar redistribution. The redistribution occurs in parallel across the subsets of cortical segments that differ in modality and layer arrangements. The recent recall induces redistribution characterized by a more complex pattern compared with redistribution induced by the remote recall. Still, the upper layers represent memory recall most persistently by the enlarged Arc mRNA fractions.

\section{TIME-AND LAYER-DEPTH INDEPENDENT INCREASE IN LEA Arc EXPRESSION}

The LEA-2 region in WMT group expressed more Arc mRNA both after recent and remote recall. The task-specific increase of Arc expression did not differ between the L6-4, L3 and L2 after both the recent $\left[F_{(2,132)}=0.67, p=0.51\right]$ and remote recall $\left[F_{(2,113)}=1.12\right.$, 


\section{Remote Memory Recall}
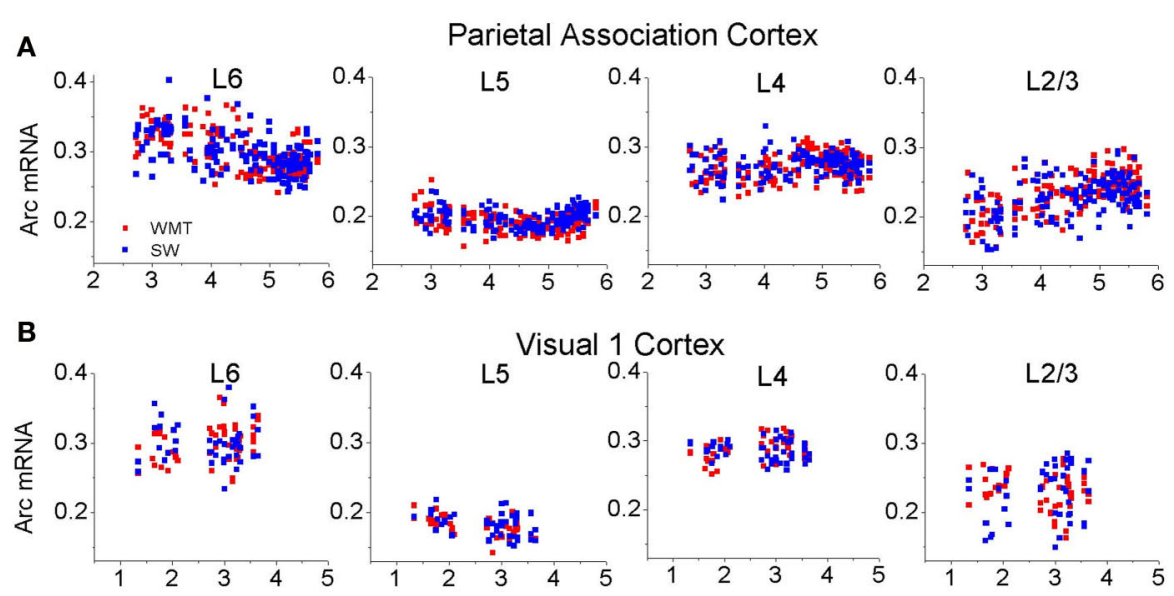

C

Motor 1 Cortex
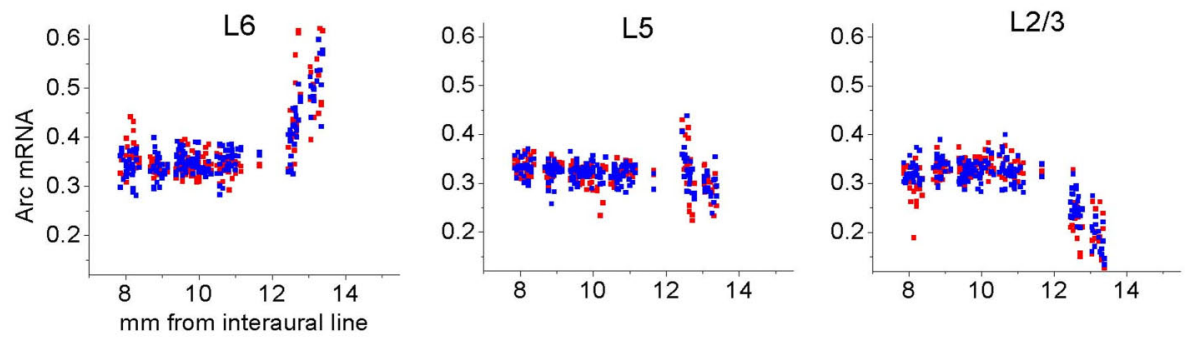

D

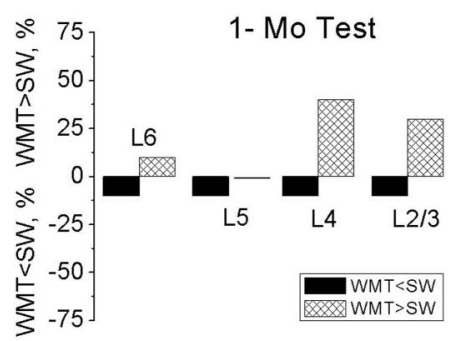

FIGURE 7 |The reorganized stereotypical pattern in Arc laminar redistribution appeared after remote WM recall. The probe tests on aWM task learned 1 month ago induced more frequent increases in Arc mRNA fractions in the upper layers $L 4$ and $L 2 / 3$ as compared to the lower layers. However the decreases in Arc fractions in L6 characteristic for recent recall became less frequent within the parietal association (A), V1 (B) and M1 (C) cortices at the 1-month interval. (D) A summary plot depicts frequencies of the segments that displayed redistribution in Arc laminar fractions after the remote memory recall. $p=0.33$; Table $S 1$ in Supplementary Material; see the LEA maps in Figure S3F in Supplementary Material]. This overall increase in Arc expression after memory recall occurred without changes in the Arc laminar distribution (Figure S5L and Table S3 in Supplementary Material).

\section{DISCUSSION}

Our main goal was to examine spatial selectivity in cortical activation during recall of recent (24-h) and remote (1-month-old) WM memory. We used large-scale mapping of Arc mRNA expression as a molecular tool to decipher regions that could be implicated in systems-level memory consolidation. Such regions may contain memory storage sites and neurons expressing mnemonic plasticity. In the present study, we report the first comparison of Arc mRNA expression in the entire cerebral cortex which allowed us to decipher elements of stereotypy in neuronal activity that accompanies memory recall. Our data suggest global principles underlying formation of cortical circuits representing the recall of remote WM memory at the levels of anatomical areas, subregions and layers.

We found that cortical anatomical areas can be subdivided into segments based on the presence of task-specific changes in neuronal activity. Remarkably, the overall levels of task-specific increase in activity and the patterns of its laminar distribution were stereotypical in those segments across the sensory, motor and associative areas. However, with memory retention, the overall increase in activity became smaller and spatially more restricted, and the laminar pattern of activity redistribution was modified. Cortical segments expressed less Arc mRNA more frequently after remote recall compared to recent recall. 


\section{SPATIAL MEMORY DOES NOT DECLINE OVER A 1-MONTH PERIOD. WATER-MAZE NAVIGATION INDUCES Arc mRNA LEVELS SPECIFIC TO THE TASK PERFORMANCE}

We did not find statistically significant differences between recent and remote memory groups in every parameter describing WM behavior (Gusev et al., 2005). These behavioral parameters included number of target crossings, target crossing latencies and proximity to the platform. WMT rats consistently swam closer to the platform location compared to the SW groups and this difference was not time-dependent. The three consecutive probe tests ameliorated possible recall difficulties at the remote time point and allowed us to access and to measure precisely stored memory.

The specificity of the retrieval-based activity in WMT rats was determined by subtraction of the activity measured in SW controls. We measured learning-specific activity by determining differences in normalized Arc mRNA levels between WMT and SW control rats following three probe tests. SW controls certainly learned and remembered the environment and the swimming experience. However, we dissected a component of the Arc signal that most likely reflects the unique navigation skills acquired, stored and reactivated by WMT rats. We also confirmed the previously observed task-specificity of the normalized Arc signal by measuring similar corticosterone levels in WMT and SW rats after the memory tests and control procedures (Gusev et al., 2005). In addition, there was no difference in the total swum distance between the WMT and SW rats in each retention group. The fact that both WMT and SW groups undergo similar and moderate changes in the swum distances over a 1-month retention interval does not affect the conclusions based on the relative Arc mRNA levels measured within each retention group (for further discussion of our behavioral protocol design, see Supplementary Material).

Thus, overall WM performance was consistent at both retrieval time points and behavioral variation does not account for the timedependent changes in relative magnitudes of the task-specific Arc mRNA expression (Gusev et al., 2005).

\section{THE STEREOTYPY IN OVERALL AND ArC LAMINAR EXPRESSION AFTER MEMORY RECALL: IMPLICATION FOR MEMORY ENGRAM SEARCH AND THEORY OF CORTICAL FUNCTION}

Arc has been related to memory consolidation and neuronal plasticity (Guzowski et al., 2000; Plath et al., 2006; Rial Verde et al., 2006), and thus, Arc expression induced by memory recall may indicate memory storage sites. The stereotypical Arc patterns representing memory recall suggest that the memory engram itself might have a stereotypical and well-ordered organization across many cortical areas. Such stereotypical organization might facilitate deciphering the engram by pointing at the most common location of putative storage sites. This could help overcome detection difficulties imposed by sparse information representation in the brain.

\section{Activated cortical segments display stereotypical increase in overall Arc mRNA levels}

We found that the majority of cortical segments displayed a taskspecific increase in Arc mRNA expression after recent recall and about half of the segments displayed an increase in Arc expression after remote memory recall. The magnitude of the overall increase in Arc expression averaged across all layers did not differ between the different segments across the entire cortex when compared within each retention group. This stereotypical elevation in Arc gene transcription occurs in putative excitatory neurons (Vazdarjanova et al., 2006) in multiple cortical areas that were previously implicated in ego- and allocentric aspects of spatial navigation including parietal association, retrosplenial, entorhinal lateral and medial areas (Save and Poucet, 2000; Harker and Whishaw, 2004; Parron et al., 2004; van Groen et al., 2004; Hafting et al., 2005; Steffenach et al., 2005; McNaughton et al., 2006). Additionally, visual 1,2M and 2L (Hoh et al., 2003; Whishaw, 2004), somatosensory 1 and 2, and motor 1 and 2 cortices also demonstrated stereotypical increases in $\operatorname{Arc}$ mRNA expression suggesting their role in the representation of WM memory recall. Increased and dynamic Arc gene expression selective to a subset of stereotypically organized cortical segments is in line with dynamic and selective activation of the human cortex over periods of memory consolidation (Rosenbaum et al., 2004; Moscovitch et al., 2005; Takashima et al., 2009; Yamashita et al., 2009).

The recall of WM spatial memory is represented not only by a stereotypical increase in overall activity level but also in stereotypical patterns of Arc laminar expression across a subset of cortical areas of different sensory modalities and hierarchy.

\section{Patterns of Arc laminar distribution differ after recent and remote recall but the upper layers represent recall by larger Arc fractions persistently}

During recent memory recall, there was a systematic bias toward the largest magnitude of task-induced activity in layers $2 / 3-4$ in multiple areas. By comparison, at the 1-month-old memory recall, the laminar distribution of weaker Arc signal was more heterogeneous, varying by layer and area. Still, Arc mRNA fractions expressed in upper layers $2 / 3-4$ increased after both recent and remote recall while layer 6 fractions decreased only after recent recall.

The stronger Arc expression in the upper layers is consistent with elevated rates of learning-induced neuronal plasticity (Miller, 1996; Rioult-Pedotti et al., 2000; Polley et al., 2004; Hodgson et al., 2005; Nichols et al., 2007) possibly mediated by higher intrinsic excitability and/or density of NMDA receptors in layers' 2/3 neurons (Monaghan and Cotman, 1985; Atzori et al., 2004). Stronger Arc expression in the upper layers may reflect an increased influence of feedback projections from the mPFC underlying processes of attention, planning and goal search (Dehaene et al., 1998; Schroeder and Foxe, 2002; Gonchar and Burkhalter, 2003; Lakatos et al., 2007) or an increased impact from the parahippocampal cortices relaying allocentric information from the hippocampus (Burke et al., 2005).

Thus, Arc laminar expression has been transformed over a1-month retention interval but the upper layers $2 / 3-4$ would be priority targets for the search of putative neuronal modifications underlying recent or remote memories.

\section{Arc expression modification and theory of modular organization of cortical signal processing}

The stereotypy in overall and layer-specific increase in Arc mRNA expression and the stereotypical redistribution in Arc laminar fractions induced by memory recall are in line with the theory of modular cortical organization. The theory proposes that some aspects of 
cortical laminar processing are common across different cortical areas (Creutzfeldt, 1977; Mountcastle, 1997; Silberberg et al., 2002; Hawkins, 2004; Hirsch and Martinez, 2006). It has been demonstrated that the circuitry of one sensory cortex can be utilized for analysis by a different sensory system after a peripheral sensory trauma (Goyal et al., 2006). Our findings of stereotypical patterns of cortical activation extend the experimental evidence and theoretical views established originally for sensory neurophysiology to memory recall for the first time.

We hypothesize that stereotypical Arc expression specific to memory recall is produced, at least in part, by the function of some basic and common algorithm of laminar processing. This putative algorithm undergoes time-dependent transformation that reflects systems-level memory consolidation within the neocortex. Stereotypy of algorithms underlying perception as well as stereotypy of algorithms underlying memory recall at various stages of memory retention would warrant production of universal activity patterns necessary for interregional cortical communication, information integration and construction of a model of the world by the neocortex (Hawkins, 2004).

\section{REMOTE MEMORY RECALL INDUCES CLUSTERED INCREASE IN ArC EXPRESSION: IMPLICATION FOR THEORY OF SYSTEMS-LEVEL CONSOLIDATION}

Systems-level memory consolidation is broadly defined as time-dependent changes in memory representation observed in various brain regions and behavioral tasks, in vertebrate as well as in invertebrate species (Setlow and McGaugh, 1999; Micheau et al., 2004; Pennartz et al., 2004; Frankland and Bontempi, 2005; Yin et al., 2009).

\section{The clustered elevation in Arc expression: only select segments are activated by both recent and remote recall}

The reorganized hippocampal and neocortical activity observed in our previous and current research accompanies the stable performance on WM task separated by a 1-month retention interval. The widespread increase in Arc expression in the neocortex and the hippocampus (Gusev et al., 2005) follows recent WM memory recall. Increases in Arc expression become clustered in both structures after remote recall. The magnitude of Arc mRNA expression induced by memory recall evolves over a 1-month retention interval in an area-specific manner. Only select areas and segments in both the neocortex and the hippocampus express similar increases in Arc expression both after recent and remote recall. Following remote recall, significant number of regions in both structures showed smaller increases in Arc expression, lack of changes in Arc expression and even less Arc expression than in SW controls.

The stable increases in Arc expression restricted to mPFC segments FrAs, Prl-1 and MO-1 following performances on both the recent and remote WM tasks support a time-independent role for mPFC in organizing memory retrieval (establishing the goals, initiating and guiding search, monitoring and verifying the memories and current experience (Granon and Poucet, 1995; Miyashita, 2004). The parietal cortex implicated in the accuracy of a search trajectory (Save and Poucet, 2000) showed a transformation in Arc gene expression over the 1-month retention interval. While the caudal parietal cortex showed more Arc expression both following the recent and remote recall, the rostral parietal cortex switched to less Arc expression at the remote recall. A persistent task-specific increase in Arc expression restricted to the caudal part of RS cortex (RS-3 segment) is consistent with specific involvement of RS-3 in WM task established by lesion studies (Vann et al., 2003).

It is noteworthy that the smaller task-related Arc expression across both the neocortex and hippocampus after remote recall is reminiscent of the overall smaller changes in neuronal activity that accompany improvement in task performance during training (Christian and Thompson, 2003; Baeg et al., 2007). Decreases in Arc mRNA expression have been observed during continued training sessions in water-maze and lever-pressing task over several days (Guzowski et al., 2001; Kelly and Deadwyler, 2002, 2003), and after learning a new environment (Ramirez-Amaya et al., 2005; Wang et al., 2006). Thus memory consolidation is accompanied by a decline in Arc expression during and after training on a scale of days and weeks.

Variation in persistence of $A r c$ mRNA induction across different brain regions was also found after training sessions in a leverpress task (Kelly and Deadwyler, 2003). Only select regions were active persistently at early and more advanced training or retention stages suggesting differential contribution of various structures to memory consolidation.

\section{The decrease in task-specific Arc signal as a possible outcome of general principles in brain function}

The overall decrease in task-related Arc expression during memory consolidation is also compatible with ideas that communication in the cortex is shaped by principles of sparse coding, energy constraints (Laughlin and Sejnowski, 2003), and homeostatic regulation of network activity (Abbott, 2003; Marder and Goaillard, 2006; Turrigiano, 2007). Thus mechanisms of systems consolidation may be shaped by these general rules of brain function. We speculate that WM memory consolidation increases efficiency of neurons participating in WM task performance: an overall lower activity volume accompanies robust recall and WM task performance at 1 month in memory retention.

\section{Comparison Arc mRNA and c-fos/zif 286 expression patterns}

The outcome of our Arc expression mapping in WMT rats differs from results obtained in mice trained on WM tasks that used visible platforms with fixed or varied positions (Teixeira et al., 2006). First, contrary to our findings of a robust Arc task-specific signal in the hippocampus (Gusev et al., 2005), there was no task-specific difference in number of c-fos positive cells in the hippocampi of mice from both recent (1-day-old) and remote (1-month-old) memory groups (Teixeira et al., 2006). Second, contrary to a diminished Arc task-specific signal in the anterior cingulate cortex after a 1-month recall, a task-specific increase in number of c-fos cells has been observed in a remote memory group in mice. We cannot exclude the possibility that an expansion of Arc signal across the entire cortex occurred at some point during the 7 days of training in rats before we started the Arc expression mapping. In addition, our findings in a WM task differ from findings in a radial maze task (Maviel et al., 2004). While both recent and remote WM recall induce enlarged Arc laminar fractions in layers $2 / 3$ and 4 , a radial maze task induces enlarged proportions of zif-268 and c-fos positive cells in layers $2 / 3$ and 4 of mice only after a 1-month but not after a 24 -h retention test (Maviel et al., 2004). 
The possible differences between rats and mice in gene expression (Snyder et al., 2009), the variety in complexity of behavioral tasks and control procedures, the non-similarities in mapping approaches (measures of signal density vs. cell numbers, in situ hybridization experiments vs. immunohistochemistry) and in neuronal specificity of the activity markers (Arc vs. $c$-fos) might all account for these discrepancies in representation of both recent and remote spatial memories across the hippocampus and neocortex.

\section{Arc expression and predictions of the standard and multiple trace models of systems-level consolidation}

The standard model of systems-level consolidation for declarative memories proposes that the neocortex predominantly or even exclusively represents and stores permanent memories (Squire, 1992; McClelland et al., 1995). Although a critical role of the neocortex for remote WM formation has been established (Frankland et al., 2001; Remondes and Schuman, 2004; Teixeira et al., 2006), its exclusiveness in remote memory storage is still widely debated for the WM task (for reference see Martin and Clark, 2007), as well as for other declarative memory tasks, due to their persistent dependence on the hippocampus (Sutherland et al., 2001; Gaskin et al., 2003; Lehmann et al., 2007).

Remote WM memory recall induced an increased Arc signal in the Prl-1 and Prl-3 segments of prefrontal cortex (mPFC) but an overall decreased Arc signal in the hippocampus when compared to recent recall. These opposite changes support a hypothesis that the mPFC overtakes (at least partly) the hippocampal role in cortical module integration during systems-level consolidation (Frankland and Bontempi, 2006; Takashima et al., 2006). The elevated difficulty of remote recall compared to recent recall known for declarative memory tasks (Biedenkapp and Rudy, 2007; Wiltgen and Silva, 2007) might also induce the restricted increase in mPFC activation for remote recall. However, such an explanation would be less likely under our protocol that we specifically designed to ameliorate recall difficulties by using three consecutive probe tests.

The multiple trace model for memory consolidation proposes that numbers of memory traces increase in both structures over the time (Nadel and Moscovitch, 1997). We measured only somatic Arc mRNA signal that may not reflect reliably numbers of activated synapses that may underlie a memory trace. Therefore, we cannot speculate how the changes in somatic Arc expression relate to this model.

Based on the spatially more restricted increase in Arc expression in the majority of cortical areas and the hippocampus induced by the robust remote memory recall, we hypothesize that systems-level memory consolidation is accompanied by enhanced functional subdivision and enhanced efficiency of the brain areas in memory activation and task performance.

Mapping, lesion and inactivation approaches alone cannot distinguish whether the hippocampus and neocortex both store remote memories or whether the hippocampus continues to be involved only in the recall and information processing functions while only the neocortex stores permanent memories (Teixeira et al., 2006). The enduring neuronal correlates for declarative memories storage (Ramirez-Amaya et al., 2001; Frankland et al., 2004; Maviel et al., 2004; Restivo et al., 2009) and the persistent although reorganized increases in Arc expression have been found in both the neocortex and hippocampus. This evidence suggests that instead of a complete transfer of memory storage into the neocortex, both structures participate in storage at some point in memory retention. A future challenging task would be to determine how recall and storage functions of these two structures evolve during systems consolidation.

\section{Arc EXPRESSION MAPS CAN HELP TO DIFFERENTIATE MEMORY RECALL AND STORAGE FUNCTIONS: A GUIDE FOR INACTIVATION AND DETECTION STUDIES}

The smaller magnitude of task-specific activity and its pronounced spatial clustering after remote recall may explain the difficulties in detection of remote memory correlates in the hippocampus and the cerebral cortex (Pascale et al., 2004; Kim and Linden, 2007; Holtmaat and Svoboda, 2009). The detailed Arc maps of putative storage sites could be helpful in making detection studies more focused and feasible. Molecular processes underlying protein-dependent spatial memory reconsolidation and an update of a stored model for the WM (Suzuki et al., 2004; Rossato et al., 2006) might be initiated in Arc expressing neurons after memory recall.

An intriguing issue of how recent and remote memories interact at the level of single neurons and synapses could be addressed more efficiently in neocortical and hippocampal segments persistently representing memory recall. Focusing on such segments may help to understand how old information is preserved during new information encoding (for the coordinates of segments with persistent and altered Arc expression, see Tables S1-S3 and Figures S3-S5 in Supplementary Material). In the future, it will be important to determine functional roles of the cortical segments that display more or less Arc expression by studying the effect of their inactivation or activation on recent and remote recall.

\section{CONCLUSION}

Our behavioral protocol allows the induction a robust remote memory and the ability to gain access to it during recall. The main trend in global reorganization of neuronal activity underlying memory recall in the cerebral cortex is parallel to changes we described previously in the hippocampus (Gusev et al., 2005). The reorganization in neocortical Arc expression indicates that systemslevel consolidation is associated with smaller overall volume and restricted spatial distribution of task-specific activity and with changes in the patterns of laminar activity during remote memory recall. Efficient recall of remote memory is accompanied by an increased functional subdivision within both the neocortex and hippocampus; robust remote task performance is achieved with fewer activated segments compared to a recent memory task.

\section{ACKNOWLEDGMENTS}

Funding for this project was provided by Blanchette Rockefeller Neurosciences Institute (BRNI). We thank Drs. Marco Atzori, M. Catherine Bennett, Rui Costa, Changhai Cui, Paul Frankland, Gudrun Ihrke, Alvin W. Lyckman, Michael J. Schell, Bernard G. Schreurs, and colleagues at BRNI for helpful advice on manuscript. We thank Madelon Cook for manuscript assistance.

\section{SUPPLEMENTARY MATERIAL}

The Supplementary Material for this article can be found online at http://www.frontiersin.org/neuroscience/integrativeneuroscience/ paper/10.3389/fnint.2010.00015/ 


\section{REFERENCES}

Abbott, L. F. (2003). Balancing homeostasis and learning in neural circuits. Zoology (Jena) 106, 365-371.

Amaral, D. G., and Witter, M. P. (1989). The three-dimensional organization of the hippocampal formation: a review of anatomical data. Neuroscience 31, 571-591.

Atzori, M., Flores, H. J., and Pineda, J. C. (2004). Interlaminar differences of spike activation threshold in the auditory cortex of the rat. Hear. Res. 189, 101-106.

Baeg, E. H., Kim, Y. B., Kim, J., Ghim, J. W., Kim, J. J., and Jung, M. W. (2007). Learning-induced enduring changes in functional connectivity among prefrontal cortical neurons. J. Neurosci. 27, 909-918.

Biedenkapp, J. C., and Rudy, J. W. (2007). Context preexposure prevents forgetting of a contextual fear memory: implication for regional changes in brain activation patterns associated with recent and remote memory tests. Learn. Mem. 14, 200-203.

Burke, S. N., Chawla, M. K., Penner, M. R., Crowell, B. E., Worley, P. F., Barnes, C. A., and McNaughton, B. L. (2005). Differential encoding of behavior and spatial context in deep and superficial layers of the neocortex. Neuron 45, 667-674.

Christian, K. M., and Thompson, R. F. (2003). Neural substrates of eyeblink conditioning: acquisition and retention. Learn. Mem. 10, 427-455.

Creutzfeldt, O. D. (1977). Generality of the functional structure of the neocortex. Naturwissenschaften 64, 507-517.

Dehaene, S., Kerszberg, M., and Changeux, J. P. (1998). A neuronal model of a global workspace in effortful cognitive tasks. Proc. Natl. Acad. Sci. U.S.A. 95, 14529-14534.

Eichenbaum, H., Stewart, C., and Morris, R. G. (1990). Hippocampal representation in place learning. J. Neurosci. 10, 3531-3542.

Frankland, P. W., and Bontempi, B. (2005). The organization of recent and remote memories. Nat. Rev. Neurosci. 6, 119-130.

Frankland, P.W., and Bontempi, B. (2006). Fast track to the medial prefrontal cortex. Proc. Natl. Acad. Sci. U.S.A.103, 509-510.

Frankland, P. W., Bontempi, B., Talton, L. E., Kaczmarek, L., and Silva, A. J. (2004). The involvement of the anterior cingulate cortex in remote contextual fear memory. Science 304, 881-883.

Frankland, P. W., O’Brien, C., Ohno, M., Kirkwood, A., and Silva, A. J. (2001). Alpha-CaMKII-dependent plasticity in the cortex is required for permanent memory. Nature 411, 309-313.

Gallagher, M., Burwell, R., and Burchinal M. (1993). Severity of spatial learning impairment in aging: development of a learning index for performance in the Morris water maze. Behav. Neurosci. 107, 618-626.

Gaskin, S., Tremblay, A., and Mumby, D. G. (2003). Retrograde and anterograde object recognition in rats with hippocampal lesions. Hippocampus 13, 962-969.

Gonchar, Y., and Burkhalter, A. (2003). Distinct GABAergic targets of feedforward and feedback connections between lower and higher areas of rat visual cortex. J. Neurosci. 23, 10904-10912.

Goyal, M. S., Hansen, P. J., and Blakemore, C. B. (2006). Tactile perception recruits functionally related visual areas in the late-blind. Neuroreport 17, 1381-1384.

Granon, S., and Poucet, B. (1995). Medial prefrontal lesions in the rat and spatial navigation: evidence for impaired planning. Behav. Neurosci. 109, 474-484.

Gusev, P. A., and Alkon, D. L. (2001) Intracellular correlates of spatial memory acquisition in hippocampal slices: long-term disinhibition of CA1 pyramidal cells. J. Neurophysiol. 86, 881-899.

Gusev, P. A., Cui, C., Alkon, D. L., and Gubin, A. N. (2005). Topography of Arc/Arg3.1 mRNA expression in the dorsal and ventral hippocampus induced by recent and remote spatial memory recall: dissociation of $\mathrm{CA} 3$ and CA1 activation. J. Neurosci. 25, 9384-9397.

Guzowski, J. F., Lyford, G. L., Stevenson, G. D., Houston, F. P., McGaugh, J. L., Worley, P. F., and Barnes, C. A. (2000). Inhibition of activity-dependent arc protein expression in the rat hippocampus impairs the maintenance of long-term potentiation and the consolidation of long-term memory. J. Neurosci. 20, 3993-4001.

Guzowski, J. F., McNaughton, B. L., Barnes, C. A., and Worley, P. F. (1999). Environment-specific expression of the immediate-early gene Arc in hippocampal neuronal ensembles. Nat. Neurosci. 2, 1120-1124.

Guzowski, J. F., Setlow, B., Wagner, E. K., and McGaugh,J.L. (2001). Experiencedependent gene expression in the rat hippocampus after spatial learning: a comparison of the immediate-early genes Arc, c-fos, and zif268. J. Neurosci. 21, 5089-5098.

Hafting, T., Fyhn, M., Molden, S., Moser, M. B., and Moser, E. I. (2005). Microstructure of a spatial map in the entorhinal cortex. Nature 436 801-806.

Harker, K. T., and Whishaw, I. Q. (2004). A reaffirmation of the retrosplenial contribution to rodent navigation: reviewing the influences of lesion, strain, and task. Neurosci. Biobehav. Rev. 28, 485-496.

Hawkins, J. B. S. (2004). On Intelligence. New York: Times Books/Henry Holt and Company, 1-261.

Hirsch, J. A., and Martinez, L. M. (2006). Laminar processing in the visual cortical column. Curr. Opin. Neurobiol. 16, 377-384.

Hodgson, R. A., Ji, Z., Standish, S., BoydHodgson, T. E., Henderson, A. K., and Racine, R. J. (2005). Training-induced and electrically induced potentiation in the neocortex. Neurobiol. Learn. Mem. 83, 22-32.

Hoh, T.E., Kolb, B., Eppel, A., Vanderwolf, C. H., and Cain, D. P. (2003). Role of the neocortex in the water maze task in the rat: a detailed behavioral and Golgi-Cox analysis. Behav. Brain Res. 138, 81-94.

Holtmaat, A., and Svoboda, K. (2009). Experience-dependent structural synaptic plasticity in the mammalian brain. Nat. Rev. Neurosci. 10, 647-658.

Kee, N., Teixeira, C. M., Wang, A. H. and Frankland, P. W. (2007). Imaging activation of adult-generated granule cells in spatial memory. Nat. Protoc. 2, 3033-3044.

Kelly, M. P., and Deadwyler, S. A. (2002) Acquisition of a novel behavior induces higher levels of Arc mRNA than does overtrained performance. Neuroscience 110, 617-626.

Kelly, M. P., and Deadwyler, S. A. (2003) Experience-dependent regulation of the immediate-early gene arc differs across brain regions. J. Neurosci. 23, 6443-6451.

Kim, S. J., and Linden, D. J. (2007) Ubiquitous plasticity and memory storage. Neuron 56, 582-592.

Kubik, S., Miyashita, T., and Guzowski, J. F. (2007). Using immediate-early genes to map hippocampal subregional functions. Learn. Mem. 14, 758-770.

Lakatos, P., Chen, C. M., O'Connell, M.N. Mills, A., and Schroeder, C. E. (2007) Neuronal oscillations and multisensory interaction in primary auditory cortex. Neuron 53, 279-292.

Laughlin, S. B., and Sejnowski, T. J. (2003). Communication in neuronal networks. Science 301, 1870-1874

Lehmann, H., Lacanilao, S., and Sutherland, R. J. (2007). Complete or partial hippocampal damage produces equivalent retrograde amnesia for remote contextual fear memories. Eur. J. Neurosci. 25, 1278-1286.
Lein, E. S., Hawrylycz, M. J., Ao, N., Ayres, M., Bensinger, A., Bernard, A., Boe, A. F., Boguski, M. S., Brockway, K. S., Byrnes, E. J., Chen, L., Chen, L., Chen, T. M., Chin, M. C., Chong, J., Crook, B. E., Czaplinska, A., Dang, C. N., Datta, S., Dee, N. R., Desaki, A. L., Desta, T., Diep, E., Dolbeare, T. A., Donelan, M. J., Dong, H. W., Dougherty, J. G., Duncan, B. J., Ebbert, A. J., Eichele, G., Estin, L. K., Faber, C., Facer, B. A., Fields, R., Fischer, S. R., Fliss, T. P., Frensley, C., Gates, S. N., Glattfelder, K. J., Halverson, K. R., Hart, M. R., Hohmann, J. G., Howell, M. P., Jeung, D. P., Johnson, R. A., Karr, P. T., Kawal, R., Kidney, J. M., Knapik, R. H., Kuan, C. L., Lake, J. H., Laramee, A. R., Larsen, K. D., Lau, C., Lemon, T. A., Liang, A. J., Liu, Y., Luong, L. T. Michaels, J., Morgan, J. J., Morgan, R. J., Mortrud, M. T., Mosqueda, N. F., Ng, L. L., Ng, R., Orta, G. J., Overly, C. C., Pak, T. H., Parry, S. E., Pathak, S. D., Pearson, O. C., Puchalski, R. B., Riley, Z. L., Rockett, H. R., Rowland, S. A., Royall, J. J., Ruiz, M. J., Sarno, N. R., Schaffnit, K., Shapovalova, N. V., Sivisay, T., Slaughterbeck, C. R., Smith, S. C., Smith, K. A., Smith, B. I., Sodt, A. J., Stewart, N. N., Stumpf, K. R., Sunkin, S. M., Sutram, M., Tam, A., Teemer, C. D., Thaller, C., Thompson, C. L., Varnam, L. R., Visel, A., Whitlock, R. M., Wohnoutka, P. E., Wolkey, C. K., Wong, V. Y., Wood, M., Yaylaoglu, M. B., Young, R. C., Youngstrom, B. L., Yuan, X. F., Zhang, B., Zwingman, T. A., and Jones, A. R. (2007). Genome-wide atlas of gene expression in the adult mouse brain. Nature 445, 168-176.

Link, W., Konietzko, U., Kauselmann, G., Krug, M., Schwanke, B., Frey, U., and Kuhl,D. (1995). Somatodendritic expression of an immediate early gene is regulated by synaptic activity. Proc. Natl. Acad. Sci. U.S.A. 92, 5734-5738.

Lyford, G. L., Yamagata, K., Kaufmann, W. E., Barnes, C. A., Sanders, L. K., Copeland, N. G., Gilbert, D. J., Jenkins, N. A., Lanahan, A. A., and Worley, P. F. (1995). Arc, a growth factor and activity-regulated gene, encodes a novel cytoskeleton-associated protein that is enriched in neuronal dendrites. Neuron 14, 433-445.

Maei, H. R., Zaslavsky, K., Teixeira, C. M., and Frankland, P. W. (2009). What is the most sensitive measure of water maze probe test performance? Front. Integr. Neurosci. 3:4. doi: 10.3389/ neuro.07.004.2009.

Marder, E., and Goaillard, J. M. (2006). Variability, compensation and homeostasis in neuron and network function. Nat. Rev. Neurosci. 7, 563-574. 
Markram, H. (2006). The blue brain project. Nat. Rev. Neurosci. 7, 153-160.

Martin, S. J., and Clark, R. E. (2007). The rodent hippocampus and spatial memory: from synapses to systems. Cell. Mol. Life Sci. 64, 401-431.

Maviel, T., Durkin, T.P., Menzaghi, F., and Bontempi, B. (2004). Sites of neocortical reorganization critical for remote spatial memory. Science 305, 96-99.

McClelland, J. L., McNaughton, B. L., and O'Reilly, R. C. (1995). Why there are complementary learning systems in the hippocampus and neocortex: insights from the successes and failures of connectionist models of learning and memory. Psychol. Rev. 102, 419-457.

McNaughton, B. L., Battaglia, F. P., Jensen, O., Moser, E. I., and Moser, M. B. (2006). Path integration and the neural basis of the 'cognitive map'. Nat. Rev. Neurosci. 7, 663-678.

Micheau, J., Riedel, G., Roloff, E. L., Inglis, J., and Morris, R. G. (2004). Reversible hippocampal inactivation partially dissociates how and where to search in the water maze. Behav. Neurosci. $118,1022-1032$.

Miller, J.A. (1991). The calibration of 35S or $32 \mathrm{P}$ with $14 \mathrm{C}$-labeled brain paste or 14C-plastic standards for quantitative autoradiography using LKB Ultrofilm or Amersham Hyperfilm. Neurosci. Lett. 121, 211-214.

Miller, R. (1996). Neural assemblies and laminar interactions in the cerebral cortex. Biol. Cybern. 75, 253-261.

Miyashita, Y. (2004). Cognitive memory: cellular and network machineries and their top-down control. Science 306, 435-440.

Monaghan, D. T., and Cotman, C. W. (1985). Distribution of N-methyl-Daspartate-sensitive L-[3H]glutamatebinding sites in rat brain. J. Neurosci. 5, 2909-2919.

Montag-Sallaz, M., and Montag, D. (2003). Learning-induced arg 3.1/arc mRNA expression in the mouse brain. Learn. Mem. 10, 99-107.

Morris, R. (1984). Developments of a water-maze procedure for studying spatial learning in the rat. J. Neurosci. Methods 11, 47-60.

Moscovitch, M., Rosenbaum, R. S., Gilboa, A., Addis, D. R., Westmacott, R., Grady, C., McAndrews, M. P., Levine, B., Black, S., Winocur, G., and Nadel, L. (2005). Functional neuroanatomy of remote episodic, semantic and spatial memory: a unified account based on multiple trace theory. J. Anat. 207, 35-66.

Mountcastle, V. B. (1997). The columnar organization of the neocortex. Brain 120(Pt 4), 701-722.
Mountcastle, V. B. (2003). Introduction. Computation in cortical columns. Cereb. Cortex 13, 2-4.

Nadel, L., and Moscovitch, M. (1997). Memory consolidation, retrograde amnesia and the hippocampal complex. Curr. Opin. Neurobiol. 7, 217-227.

Nichols, J. A., Jakkamsetti, V. P., Salgado, H., Dinh, L., Kilgard, M. P., and Atzori, M. (2007). Environmental enrichment selectively increases glutamatergic responses in layer II/III of the auditory cortex of the rat. Neuroscience $145,832-840$.

Parron, C., Poucet, B., and Save, E. (2004). Entorhinal cortex lesions impair the use of distal but not proximal landmarks during place navigation in the rat. Behav. Brain Res. 154, 345-352.

Pascale, A., Gusev, P. A., Amadio, M., Dottorini, T., Govoni, S., Alkon, D. L. and Quattrone, A. (2004). Increase of the RNA-binding protein $\mathrm{HuD}$ and posttranscriptional up-regulation of the GAP-43 gene during spatial memory. Proc. Natl. Acad. Sci. U.S.A. 101, 1217-1222.

Paxinos, G., and Watson, C. (1998). The Rat Brain in Stereotaxic Coordinates. London, UK/San Diego, CA, USA: Academic Press Limited.

Pennartz, C. M., Lee, E., Verheul, J., Lipa, P., Barnes, C. A., and McNaughton, B. L. (2004). The ventral striatum in off-line processing: ensemble reactivation during sleep and modulation by hippocampal ripples. J. Neurosci. 24, 6446-6456.

Plath, N., Ohana, O., Dammermann, B., Errington, M. L., Schmitz, D., Gross, C., Mao, X., Engelsberg, A., Mahlke, C., Welzl, H., Kobalz, U., Stawrakakis, A., Fernandez, E., Waltereit, R., BickSander, A., Therstappen, E., Cooke, S. F., Blanquet, V., Wurst, W., Salmen, B., Bosl, M. R., Lipp, H. P., Grant, S. G., Bliss, T. V., Wolfer, D. P., and Kuhl, D. (2006). Arc/Arg3.1 is essential for the consolidation of synaptic plasticity and memories. Neuron 52, 437-444.

Polley, D. B., Kvasnak, E., and Frostig, R. D. (2004). Naturalistic experience transforms sensory maps in the adult cortex of caged animals. Nature 429, 67-71.

Ramirez-Amaya, V., Vazdarjanova, A., Mikhael, D., Rosi, S., Worley, P. F., and Barnes, C. A. (2005). Spatial exploration-induced Arc mRNA and protein expression: evidence for selective, network-specific reactivation. J. Neurosci. 25, 1761-1768.

Ramirez-Amaya, V., Balderas, I., Sandoval, J., Escobar, M. L., and BermudezRattoni, F. (2001). Spatial longterm memory is related to mossy fiber synaptogenesis. J. Neurosci. 21, 7340-7348.
Remondes, M., and Schuman, E. M. (2004). Role for a cortical input to hippocampal area CA1 in the consolidation of a long-term memory. Nature 431, 699-703.

Restivo, L., Vetere, G., Bontempi, B., and mmassari-Teule, M. (2009). The formation of recent and remote memory is associated with time-dependent formation of dendritic spines in the hippocampus and anterior cingulate cortex. J. Neurosci. 29, 8206-8214.

Rial Verde, E. M., Lee-Osbourne, J., Worley, P. F., Malinow, R., and Cline, H. T. (2006). Increased expression of the immediate-early gene arc/arg3.1 reduces AMPA receptor-mediated synaptic transmission. Neuron 52 461-474.

Rioult-Pedotti, M. S., Friedman, D., and Donoghue, J. P. (2000). Learninginduced LTP in neocortex. Science 290, 533-536.

Rosenbaum, R. S., Ziegler, M., Winocur, G., Grady, C. L., and Moscovitch, M. (2004). "I have often walked down this street before": fMRI studies on the hippocampus and other structures during mental navigation of an old environment. Hippocampus 14 826-835.

Rossato, J. I., Bevilaqua, L. R., Medina, J. H., Izquierdo, I., and Cammarota, M. (2006). Retrieval induces hippocampal-dependent reconsolidation of spatial memory. Learn. Mem 13, 431-440.

Save,E., and Poucet,B. (2000).Involvement of the hippocampus and associative parietal cortex in the use of proximal and distal landmarks for navigation. Behav. Brain Res. 109, 195-206.

Schroeder, C. E., and Foxe, J.J. (2002). The timing and laminar profile of converging inputs to multisensory areas of the macaque neocortex. Brain Res. Cogn Brain Res. 14, 187-198.

Setlow, B., and McGaugh, J. L. (1999). Involvement of the posteroventral caudate-putamen in memory consolidation in the Morris water maze. Neurobiol. Learn. Mem. 71, 240-247.

Silberberg, G., Gupta, A., and Markram, H. (2002). Stereotypy in neocortical microcircuits. Trends Neurosci. 25 , 227-230.

Snyder, J. S., Choe, J. S., Clifford, M. A., Jeurling, S. I., Hurley, P., Brown, A., Kamhi, J. F., and Cameron, $\mathrm{H}$ A. (2009). Adult-born hippocampal neurons are more numerous, faster maturing, and more involved in behavior in rats than in mice. $J$. Neurosci. 29, 14484-14495.

Squire, L. R. (1992). Memory and the hippocampus: a synthesis from findings with rats, monkeys, and humans. Psychol. Rev. 99, 195-231.
Squire, L. R., and Bayley, P. J. (2007). The neuroscience of remote memory. Curr. Opin. Neurobiol. 17, 185-196.

Steffenach, H.A., Witter, M., Moser, M.B., and Moser, E. I. (2005). Spatial memory in the rat requires the dorsolateral band of the entorhinal cortex. Neuron $45,301-313$.

Sutherland, R. J., Weisend, M. P., Mumby, D., Astur, R. S., Hanlon, F. M., Koerner, A., Thomas, M. J., Wu, Y., Moses, S. N., Cole, C., Hamilton, D. A., and Hoesing, J. M. (2001). Retrograde amnesia after hippocampal damage: recent vs. remote memories in two tasks. Hippocampus 11, 27-42.

Suzuki, A., Josselyn, S. A., Frankland, P.W., Masushige, S., Silva, A. J., and Kida, S. (2004). Memory reconsolidation and extinction have distinct temporal and biochemical signatures. J. Neurosci. 24 , 4787-4795.

Swanson, L. W. (2004). Brain Maps: Structure of the Rat Brain. Amsterdam: Elsevier Academic Press, 1-215.

Tagawa, Y., Kanold, P. O., Majdan, M., and Shatz, C. J. (2005). Multiple periods of functional ocular dominance plasticity in mouse visual cortex. Nat. Neurosci. 8, 380-388.

Takashima, A., Nieuwenhuis, I. L., Jensen, O., Talamini, L. M., Rijpkema, M., and Fernandez, G. (2009). Shift from hippocampal to neocortical centered retrieval network with consolidation. J. Neurosci. 29, 10087-10093.

Takashima, A., Petersson, K. M., Rutters, F., Tendolkar, I., Jensen, O., Zwarts, M. J., McNaughton, B. L., and Fernandez, G. (2006). Declarative memory consolidation in humans: a prospective functional magnetic resonance imaging study. Proc. Natl. Acad. Sci. U.S.A. 103, 756-761.

Tanaka, D., Nakada, K., Takao, K., Ogasawara, E., Kasahara, A., Sato, A., Yonekawa, H., Miyakawa, T., and Hayashi, J. (2008). Normal mitochondrial respiratory function is essential for spatial remote memory in mice. Mol. Brain 1, 21

Teixeira, C. M., Pomedli, S. R., Maei, H. R., Kee, N., and Frankland, P. W (2006). Involvement of the anterior cingulate cortex in the expression of remote spatial memory. J. Neurosci. 26, 7555-7564.

Thomson, A. M., and Bannister, A. P. (2003). Interlaminar connections in the neocortex. Cereb. Cortex 13 $5-14$

Turrigiano, G. (2007). Homeostatic signaling: the positive side of negative feedback. Curr. Opin. Neurobiol. 17, 318-324.

van Groen, T., Kadish, I., and Wyss, J. M. (2004). Retrosplenial cortex lesions of area Rgb (but not of area Rga) 
impair spatial learning and memory in the rat. Behav. Brain Res. 154, 483-491.

Vann, S. D., Kristina Wilton, L. A., Muir, J. L., and Aggleton, J. P. (2003). Testing the importance of the caudal retrosplenial cortex for spatial memory in rats. Behav. Brain Res. 140, 107-118.

Vazdarjanova, A., Ramirez-Amaya, V., Insel, N., Plummer, T. K., Rosi, S., Chowdhury, S., Mikhael, D., Worley, P. F., Guzowski, J. F., and Barnes, C. A. (2006). Spatial exploration induces ARC, a plasticity-related immediate-early gene, only in calcium/calmodulin-dependent protein kinase II-positive principal excitatory and inhibitory neurons of the rat forebrain. J. Comp. Neurol. 498, 317-329.

Wang, K. H., Majewska, A., Schummers, J., Farley, B., Hu, C., Sur, M., and Tonegawa, S. (2006). In vivo twophoton imaging reveals a role of arc in enhancing orientation specificity in visual cortex. Cell 126, 389-402.

Waydo, S., Kraskov, A., Quian, Q. R., Fried, I., and Koch, C. (2006). Sparse representation in the human medial temporal lobe. J. Neurosci. 26, 10232-10234.

Whishaw, I. Q. (2004). Posterior neocortical (visual cortex) lesions in the rat impair matching-to-place navigation in a swimming pool: a reevaluation of cortical contributions to spatial behavior using a new assessment of spatial versus non-spatial behavior. Behav. Brain Res. 155, 177-184.

Wiltgen, B. J., and Silva, A. J. (2007). Memory for context becomes less specific with time. Learn. Mem. 14, 313-317.

Xu, T., Yu, X., Perlik, A. J., Tobin, W. F., Zweig, J.A., Tennant, K., Jones, T., and Zuo, Y. (2009). Rapid formation and selective stabilization of synapses for enduring motor memories. Nature 462, 915-919.
Yamashita, K., Hirose, S., Kunimatsu, A., Aoki, S., Chikazoe, J., Jimura, K., Masutani, Y., Abe, O., Ohtomo, K., Miyashita, Y., and Konishi, S. (2009). Formation of long-term memory representation in human temporal cortex related to pictorial paired associates. $J$. Neurosci. 29, 10335-10340.

Yang, G., Pan, F., and Gan, W. B. (2009). Stably maintained dendritic spines are associated with lifelong memories. Nature 462, 920-924.

Yin, H. H., Mulcare, S. P., Hilario, M. R., Clouse, E., Holloway, T., Davis, M. I., Hansson, A. C., Lovinger, D. M., and Costa, R. M. (2009). Dynamic reorganization of striatal circuits during the acquisition and consolidation of a skill. Nat. Neurosci. 12, 333-341.

Zhang, W. P., Guzowski, J. F., and Thomas, S. A. (2005). Mapping neuronal activation and the influence of adrenergic signaling during contextual memory retrieval. Learn. Mem. 12, 239-247.
Conflict of Interest Statement: The authors declare that the research was conducted in the absence of any commercial or financial relationships that could be construed as a potential conflict of interest.

Received: 06 February 2010; paper pending published: 04 March 2010; accepted: 23 April 2010; published online: 21 May 2010.

Citation: Gusev PA and Gubin AN (2010) Arc/Arg3.1 mRNA global expression patterns elicited by memory recall in cerebral cortex differ for remote versus recent spatial memories. Front. Integr. Neurosci. 4:15. doi: 10.3389/fnint.2010.00015

Copyright (C) 2010 Gusev and Gubin. This is an open-access article subject to an exclusive license agreement between the authors and the Frontiers Research Foundation, which permits unrestricted use, distribution, and reproduction in any medium, provided the original authors and source are credited. 